\title{
APPROXIMATE CLOAKING FOR THE FULL WAVE EQUATION VIA CHANGE OF VARIABLES*
}

\author{
HOAI-MINH NGUYEN ${ }^{\dagger}$ AND MICHAEL S. VOGELIUS $\ddagger$
}

\begin{abstract}
We study, in the context of the full wave equation, an approximate cloaking scheme that was previously considered for the Helmholtz equation [R. V. Kohn, D. Onofrei, M. S. Vogelius, and M. I. Weinstein, Comm. Pure Appl. Math., 63 (2010), pp. 973-1016, H.-M. Nguyen and M. S. Vogelius, Arch. Ration. Mech. Anal., 203 (2011), pp. 769-807]. This cloaking scheme consists in a combination of an absorbing layer with an anisotropic layer, obtained by so-called transformation optics. We give optimal bounds for the visibility that tend to zero as a certain regularization parameter approaches 0 . Our bounds are based on recent estimates for the Helmholtz equation [from Nguyen and Vogelius], some low frequency improvements of these estimates, and the use of Fourier transformation in time.
\end{abstract}

Key words. wave equation, approximate cloaking, metamaterials

AMS subject classifications. 35L05, 35J05, 35R30, 78A46

DOI. $10.1137 / 110833154$

1. Introduction and statement of main results. Cloaking via change of variables (sometimes referred to as "cloaking by mapping") has received quite a bit of attention since the 2006 papers by Pendry, Schurig, and Smith [18] and Leonhardt [11]. Pendry, Schurig, and Smith approached the problem in terms of the Helmholtz equation (describing monochromatic light), whereas Leonhardt took the "ray-optics" approach. In both cases the fundamental idea was to use a singular change of variables to create a cloaked region from a single point. This idea had already been used in 2003 by Greenleaf, Lassas, and Uhlmann to generate extreme examples of nonuniqueness for the zero frequency Helmholtz inverse coefficient problem, the so-called Calderon problem [5].

In the case of perfect cloaking the objective is to construct a region (the cloaked region) in which the fields trivialize in such a way that they are completely insensitive to changes in the coefficients inside this region. Furthermore, the presence of the cloak (the "shield" that surrounds the cloaked region) should not perturbe the fields outside the cloak. The need for very singular (and anisotropic) materials not only is the primary practical difficulty but is also at the very heart of the theoretical difficulties of this cloaking problem. The main theoretical task is to define and analyze the properties of the appropriate notion of a weak (and physical) solution [2], [22]. To avoid the use of singular materials, regularized schemes have been proposed in [1], [3], [9], [19], [20], [24]. The trade-off is that one no longer attains perfect cloaking but only approximate cloaking. The approach originated in [9] appears particularly well-suited for rigorous estimation of the degree of approximate cloaking (near-invisibility). In this approach the regularization parameter $\varepsilon>0$ represents the diameter of a small ball that is mapped to the (approximately) cloaked region by a change of variables. When $\varepsilon$ approaches zero, one reaches the singular situation of a point being mapped to

\footnotetext{
*Received by the editors May 5, 2011; accepted for publication (in revised form) December 20, 2011; published electronically May 29, 2012.

http://www.siam.org/journals/sima/44-3/83315.html

${ }^{\dagger}$ Courant Institute, NYU, New York, NY 10012 (hoaiminh@cims.nyu.edu).

${ }^{\ddagger}$ Department of Mathematics, Rutgers, The State University of New Jersey, New Brunswick, NJ 08903 (vogelius@math.rutgers.edu). This author was partially supported by NSF grant DMS0604999 .
}

1894 
the (perfectly) cloaked region. The reader may find more information and references related to cloaking in the works mentioned above and in the review articles [4] and [23].

Let us briefly review some facts about approximate cloaking for the Helmholtz equation for a finite range and for the full range of frequencies. In order to succesfully achieve approximate cloaking it is often advantageous to introduce a lossy layer in addition to the standard (mapped) cloak. Using an appropriate lossy layer, it is proved in [8] that approximate cloaking works well on a bounded domain regardless of the contents of the cloaked region. In [12], the author proved that approximate cloaking works well for exterior problems with a zero Dirichlet boundary condition. In [15], the author established that approximate cloaking works well in the whole space regardless of the contents of the cloaked region (using a fixed lossy layer). The result in [12] is very related to the results in [15], since a highly conducting media (as in a lossy layer) enforces the zero Dirichlet boundary condition approximately (see, e.g., [6]). In both [8] and [15] the authors demonstrated the necessity of the lossy layer in order to obtain a degree of approximate cloaking (near-invisibility) that is independent of the contents of the cloaked region. The paper [17] establishes precise estimates for the degree of near-invisibility at all frequencies, where the dependence on frequency is explicit. These estimates are sharp and independent of the contents of the cloaked region. To be a little more specific, in the high frequency case, our "lossy" approximate cloaking scheme works as well as in the finite frequency case. However, the estimates degenerate as frequency tends to 0 . This follows from (or can be explained by) the fact that the effect of the lossy layer becomes weaker and weaker, as frequency tends to 0 . Without a lossy layer the situation becomes quite complicated, as explored in [16]. For example, in the three-dimensional (3d) nonresonant case, i.e., when $k^{2}$ is not an eigenvalue of the Neumann problem inside the cloaked region (here $k$ denotes the wave number), the approximate scheme works well: cloaking is achieved (as the parameter of regularization goes to zero) and the limiting field inside the cloaked region is the corresponding solution to the Neumann problem. In the $3 \mathrm{~d}$ resonant case, the situation changes completely. Sometimes cloaking is achieved; nevertheless, the limiting field inside the cloaked region depends on the solution in the free space. Sometimes cloaking is not achieved, and the energy inside the cloaked region tends to infinity as the parameter of regularization tends to 0 . In the two-dimensional (2d) nonresonant case, the limiting field inside the cloaked region inherits a nonlocal structure. In the $2 \mathrm{~d}$ resonant case, cloaking sometimes is not achieved, and the energy inside the cloaked region can go to infinity. These "lossless" facts are somewhat different from what is frequently asserted in the literature, namely, that (a) in three dimensions, cloaking is always achieved, the limiting field inside and outside the cloaked region separate completely, and the energy of the field inside the cloaked region remains bounded, and $(b)$ in $2 \mathrm{~d}$, the limiting field inside the cloaked region satisfies the corresponding Neumann problem.

The goal of this paper is to study approximate cloaking for the wave equation via change of variables. Although approximate cloaking has been extensively investigated for the Helmholtz equation, this is, to the best of our knowledge, the first work for the full wave equation. In our approximate cloaking scheme, we use again two layers. One comes from the standard scheme introduced in the work of Kohn et al. [9]. The other is an appropriate lossy layer, similar to what has been used for the Helmholtz equation in [8], [17]. We estimate the degree of approximate cloaking (near-invisibility) in two and three dimensions. Our results assert that the visibility is of order $\varepsilon$ in three dimensions (Theorem 1) and of the order $1 /|\ln \varepsilon|$ in two dimensions (Theorem 2). We emphasize that our estimates hold for an arbitrary finite range of material parameters 
inside the cloaked region, but that the constants depend on this range (and only on this range). We also note that this dependence of the constants on the range is real and totally consistent with the fact that the uniformly valid estimates of the degree of near-invisibility for the Helmholtz equation degenerate as frequency goes to 0 .

To obtain a wave equation estimate of the degree of near-invisibility we, briefly described, proceed as follows. We first transform the wave equation into a family of Helmholtz equations by taking the Fourier transform with respect to time. After obtaining the appropriate degree of near-invisibility estimates for the Helmholtz equation, where the dependence on frequency is explicit, we simply invert the Fourier transform. For the high frequency regime we can directly use the estimates of the degree of near-invisibility established in [17], but for the low frequency Helmholtz equation we have to establish new estimates (in section 2.2) which improve the ones in [17] under the (additional) finite range assumption. The proof of these new estimates are among the central results in this paper. We emphasize here that the estimates for the Helmholtz equation blow up as frequency goes to 0. However, they blow up in an integrable way thanks to the new estimates in section 2.2. Another important (albeit technical) point is that we need to establish that the Fourier transform of solutions to the wave equation (with respect to time) satisfy an outgoing radiation condition. The proof of this fact is contained in Appendix A.

Our analysis differs significantly between two dimensions and three dimensions. In three dimensions, we rely on Huyghens' principle to pass from the wave equation to a family of Helmholtz equations and to obtain appropriate estimates for the data of these equations. In two dimensions, we have a priori to establish sufficient decay of solutions to the wave equation at infinity in order to carry out a similar analysis. The required decay estimate is found in Appendix B. Furthermore, the $2 \mathrm{~d}$ analysis of the low frequency Helmholtz equation is considerably more delicate than the $3 \mathrm{~d}$ analogue, due to the nonuniqueness of "physical" solutions to the homogeneous Laplace equation (the zero frequency limit) in all of space (see Lemma 4).

We now state our main results precisely. For simplicity suppose the cloak occupies the annular region $\{1 / 2<|x|<2\}$ and that the cloaked region is the ball $B_{1 / 2}=$ $\{|x|<1 / 2\}$ of $\mathbb{R}^{d}(d=2,3)$. Let $F_{\varepsilon}$ denote the radial Lipschitz map, which transforms the ball $B_{\varepsilon}$ into $B_{1}$, maps $B_{2}$ onto itself, and which is given by

$$
F_{\varepsilon}(x)=\left\{\begin{array}{cl}
x & \text { if } x \in \mathbb{R}^{d} \backslash B_{2}, \\
\left(\frac{2-2 \varepsilon}{2-\varepsilon}+\frac{|x|}{2-\varepsilon}\right) \frac{x}{|x|} & \text { if } x \in B_{2} \backslash B_{\varepsilon}, \\
\frac{x}{\varepsilon} & \text { if } x \in B_{\varepsilon} .
\end{array}\right.
$$

We shall use the standard notation

$$
F_{*} A(y)=\frac{D F(x) A(x) D F^{T}(x)}{\operatorname{det} D F(x)} \quad \text { and } \quad F_{*} \Sigma(y)=\frac{\Sigma(x)}{\operatorname{det} D F(x)} \quad \text { with } x=F^{-1}(y)
$$

for any real, symmetric matrix valued function $A$ and any complex function $\Sigma$.

Let $u$ and $u_{c}$ be the unique solution to the wave equation

$$
\begin{cases}\partial_{t t}^{2} u-\Delta u=f & \text { in } \mathbb{R}_{+} \times \mathbb{R}^{d} \\ u(t=0)=u_{0} & \text { in } \mathbb{R}^{d} \\ \partial_{t} u(t=0)=u_{1} & \text { in } \mathbb{R}^{d}\end{cases}
$$


and to the "damped" wave equation

$$
\begin{cases}\Sigma_{1, c} \partial_{t t}^{2} u_{c}-\operatorname{div}\left(A_{c} \nabla u_{c}\right)+\Sigma_{2, c} \partial_{t} u_{c}=f & \text { in } \mathbb{R}_{+} \times \mathbb{R}^{d}, \\ u_{c}(t=0)=u_{0} & \text { in } \mathbb{R}^{d}, \\ \partial_{t} u_{c}(t=0)=u_{1} & \text { in } \mathbb{R}^{d},\end{cases}
$$

respectively. Here $A_{c}, \Sigma_{1, c}$, and $\Sigma_{2, c}$ are time independent and defined as

$$
A_{c}, \Sigma_{1, c}, \Sigma_{2, c}=\left\{\begin{array}{cl}
I, 1,0 & \text { in } \mathbb{R}^{d} \backslash B_{2}, \\
F_{\varepsilon *} I, F_{\varepsilon *} 1,0 & \text { in } B_{2} \backslash B_{1}, \\
F_{\varepsilon *} I, F_{\varepsilon *} 1, F_{\varepsilon *}\left(1 / \varepsilon^{2+\gamma}\right) & \text { in } B_{1} \backslash B_{1 / 2}, \\
a, \sigma, 0 & \text { in } B_{1 / 2}
\end{array}\right.
$$

for some positive constant $\gamma$ (a parameter of our scheme). Note that damping is only present in the annulus $B_{1} \backslash B_{1 / 2} .{ }^{1}$ We assume that the real, symmetric matrix valued function $a$ and the real-valued function $\sigma$ each has a "finite range" in the sense that

$$
\frac{1}{\Lambda}|\xi|^{2} \leq\langle a \xi, \xi\rangle \leq \Lambda|\xi|^{2}, \quad \frac{1}{\Lambda} \leq \sigma \leq \Lambda
$$

for some positive constant $\Lambda$.

Remark 1. Notice that to cloak the region $B_{1 / 2}$, we use two layers in the region $B_{2} \backslash B_{1 / 2}$. The first layer, in the region $B_{2} \backslash B_{1}$, is the standard "mapped cloak" introduced in [9]. The second layer, in the region $B_{1} \backslash B_{1 / 2}$, is a lossy (damping) layer used in [8], [17].

The main results of this paper are the following two theorems.

TheOrem 1. Suppose $d=3$ and $\gamma>0$. Suppose $f$ is a smooth function defined on $\mathbb{R}_{+} \times \mathbb{R}^{3}$ and suppose $u_{0}, u_{1}$ are smooth functions defined on $\mathbb{R}^{3}$ with $\operatorname{supp} f \subset$ $[0,1] \times\left(B_{4} \backslash B_{2}\right)$ and $\operatorname{supp} u_{0}, \operatorname{supp} u_{1} \subset B_{4} \backslash B_{2}$. Let $u$ and $u_{c}$ denote the unique solutions to (1.3) and (1.4), respectively, where $a$ and $\sigma$ satisfy (1.5). Given any $R>2$, there exists a positive constant $C$, depending only on the range-constant $\Lambda$, the constant $\gamma$, and $R$, such that

$$
\sup _{t>0}\left\|u_{c}(t, \cdot)-u(t, \cdot)\right\|_{L^{2}\left(B_{R} \backslash B_{2}\right)} \leq C\left(\|f\|+\left\|u_{0}\right\|+\left\|u_{1}\right\|\right) \varepsilon .
$$

Here

$$
\|f\|=\|f\|_{C^{m}}, \quad\left\|u_{0}\right\|=\left\|u_{0}\right\|_{C^{m}}, \quad \text { and } \quad\left\|u_{1}\right\|=\left\|u_{1}\right\|_{C^{m}}
$$

for some $m>0$.

TheOREm 2. Suppose $d=2$ and $\gamma>0$. Suppose $f$ is a smooth function defined on $\mathbb{R}_{+} \times \mathbb{R}^{2}$ and suppose $u_{0}, u_{1}$ are smooth functions defined on $\mathbb{R}^{2}$ with supp $f \subset$ $[0,1] \times\left(B_{4} \backslash B_{2}\right)$ and $\operatorname{supp} u_{0}, \operatorname{supp} u_{1} \subset B_{4} \backslash B_{2}$. Let $u$ and $u_{c}$ denote the unique solutions to (1.3) and (1.4), respectively, where $a$ and $\sigma$ satisfy (1.5). Given any $R>2$, there exists a positive constant $C$, depending only on the range-constant $\Lambda$, the constant $\gamma$, and $R$, such that

$$
\sup _{t>0}\left\|u_{c}(t, \cdot)-u(t, \cdot)\right\|_{L^{2}\left(B_{R} \backslash B_{2}\right)} \leq C\left(\|f\|+\left\|u_{0}\right\|+\left\|u_{1}\right\|\right) \frac{1}{|\ln \varepsilon|} .
$$

\footnotetext{
${ }^{1}$ Our analysis immediately extends to the case where the triplet $(a, \sigma, 0)$ is replaced by $\left(a, \sigma_{1}, \sigma_{2}\right)$ with $\left(a, \sigma_{1}\right)$ satisfying the same condition as $(a, \sigma)$, and $0 \leq \sigma_{2} \leq \Lambda$.
}

Copyright $\odot$ by SIAM. Unauthorized reproduction of this article is prohibited. 
Here

$$
\|f\|=\|f\|_{C^{m}}, \quad\left\|u_{0}\right\|=\left\|u_{0}\right\|_{C^{m}}, \quad \text { and } \quad\left\|u_{1}\right\|=\left\|u_{1}\right\|_{C^{m}}
$$

for some $m>0$.

Remark 2. The estimates in Theorems 1 and 2 are sharp (in the regularization parameter $\varepsilon$ ) since the minimal visibility for the Helmholtz equation is of order $\varepsilon$ in three dimensions and of order $1 /|\ln \varepsilon|$ in two dimensions in the finite frequency regime.

2. Preliminaries. As mentioned in the introduction, to obtain the degree of near-invisibility estimates for the wave equation, we first transform the wave equations into a family of Helmholtz equations and establish the appropriate degree of near-invisibility estimates for these Helmholtz equations, where the dependence on frequency is explicit. To this end, we recall some known results from the work of Morawetz and Ludwig [13], Nguyen [15, 16], and Nguyen and Vogelius [17], and then we establish new results, which will be used in the proof of Theorems 1 and 2 .

2.1. Some known results. We first recall two results concerning exterior problems. The first one, dealing with the high frequency regime, is very related to results of Morawetz and Ludwig [13] and can be proved in the same fashion as [17, Proposition 1]. In the following, whenever we talk about outgoing solutions to an exterior Helmholtz problem at frequency $k$, we mean solutions that satisfy

$$
\partial_{r} v-i k v=o\left(r^{-\frac{d-1}{2}}\right) \quad \text { as } r \text { goes to infinity . }
$$

Proposition 1. Let $d=2,3$ and $k>k_{0}$ for some $k_{0}>0$. Let $g \in H^{1}\left(\partial B_{1}\right)$ and let $v \in H_{\text {loc }}^{1}\left(\mathbb{R}^{d} \backslash B_{1}\right)$ be the unique outgoing solution of

$$
\left\{\begin{array}{l}
\Delta v+k^{2} v=0 \text { in } \mathbb{R}^{d} \backslash B_{1}, \\
v=g \text { on } \partial B_{1} .
\end{array}\right.
$$

Then

$$
\frac{1}{\beta} \int_{B_{\beta} \backslash B_{1}}\left(|\nabla v|^{2}+k^{2}|v|^{2}\right) \leq C\|g\|_{H^{1}\left(\partial B_{1}\right)}^{2}
$$

for some constant $C$ depending only on $k_{0}$.

Remark 3. Proposition 1 also holds if the unit ball $B_{1}$ is replaced by a smooth, bounded convex domain of $\mathbb{R}^{d}$. and 4].

The second result, concerning the low frequency regime, is from [16, Lemmas 1

Proposition 2. Let $d=2,3$ and $0<\varepsilon<1$. Let $D \subset B_{1}$ be a smooth, nonempty open subset of $\mathbb{R}^{d}$ and $g_{\varepsilon} \in H^{\frac{1}{2}}(\partial D)$. Assume $\mathbb{R}^{d} \backslash \bar{D}$ is connected and $v_{\varepsilon} \in H_{\text {loc }}^{1}\left(\mathbb{R}^{d} \backslash D\right)$ is the unique outgoing solution of

$$
\begin{cases}\Delta v_{\varepsilon}+\varepsilon^{2} v_{\varepsilon}=0 & \text { in } \mathbb{R}^{d} \backslash \bar{D}, \\ v_{\varepsilon}=g_{\varepsilon} & \text { on } \partial D .\end{cases}
$$

(i) We have

$$
\left\|v_{\varepsilon}\right\|_{H^{1}\left(B_{R} \backslash D\right)} \leq C_{R}\left\|g_{\varepsilon}\right\|_{H^{\frac{1}{2}}(\partial D)} \quad \forall R>1
$$

Copyright $@$ by SIAM. Unauthorized reproduction of this article is prohibited. 
and for all $\beta>1$,

$$
\begin{cases}\left\|v_{\varepsilon}\right\|_{L^{2}\left(B_{2 \beta} \backslash B_{\beta}\right)} \leq C \beta^{\frac{1}{2}}\left\|g_{\varepsilon}\right\|_{H^{\frac{1}{2}}(\partial D)} & \text { if } d=3, \\ \left\|v_{\varepsilon}\right\|_{L^{2}\left(B_{2 \beta} \backslash B_{\beta}\right)} \leq C \beta \frac{\left|H_{0}^{(1)}(\varepsilon \beta)\right|}{\left|H_{0}^{(1)}(\varepsilon)\right|}\left\|g_{\varepsilon}\right\|_{H^{\frac{1}{2}}(\partial D)} & \text { if } d=2\end{cases}
$$

for some positive constants $C_{R}=C(R, D)$ and $C=C(D)$.

(ii) Assume in addition that $g_{\varepsilon}$ converges to $g$ weakly in $H^{\frac{1}{2}}(\partial D)$ as $\varepsilon \rightarrow 0$. Then $v_{\varepsilon}$ converges to $v$ weakly in $H_{\text {loc }}^{1}\left(\mathbb{R}^{d} \backslash D\right)$, where $v \in W^{1}\left(\mathbb{R}^{d} \backslash D\right)$ is the unique solution of

$$
\begin{cases}\Delta v=0 & \text { in } \mathbb{R}^{d} \backslash \bar{D}, \\ v=g & \text { on } \partial D .\end{cases}
$$

Here and in the following, $H_{0}^{(1)}$ denotes the Hankel function of the first kind of order 0 . For a connected, smooth open region $U$ of $\mathbb{R}^{d}$ with a bounded complement (this includes $U=\mathbb{R}^{d}$ ), $W^{1}(U)$ is defined as follows:

$$
W^{1}(U)=\left\{\psi \in L_{l o c}^{2}(U) ; \quad \frac{\psi(x)}{\sqrt{1+|x|^{2}}} \in L^{2}(U) \text { and } \nabla \psi \in L^{2}(U)\right\} \quad \text { for } d=3,
$$

and

$$
W^{1}(U)=\left\{\psi \in L_{l o c}^{2}(U): \frac{\psi(x)}{\ln (2+|x|) \sqrt{1+|x|^{2}}} \in L^{2}(U) \text { and } \nabla \psi \in L^{2}(U)\right\} \quad \text { for } d=2 \text {. }
$$

Remark 4. The estimates in (2.2) are not stated explicitly in [16, Lemmas 1 and 4]. However, their proofs follow immediately from the ones of [16, Lemmas 1 and 4]. We can also view (2.2) as a limiting case of Lemma 1 below (which is [17, Theorem 1]) as $\lambda \rightarrow 0$. In fact (2.1) and (2.2), with $\beta=1 / \varepsilon$, already appeared in [15]. It is not essential that $D \subset B_{1}$; the analogue of Proposition 2 holds for $D \subset B_{R_{0}}$ with the obvious change in the range of $R$ and $\beta$.

The following lemma, which will be used in the proof of Proposition 3, was established in [17, Theorem 1].

Lemma 1. Let $d=2,3,0<\lambda<1$, and $k>0$. Let a be a real, symmetric matrix valued function, and let $\sigma$ be a complex function, both defined on $B_{1 / 2}$. Suppose $a$ is bounded and uniformly elliptic, and suppose $\sigma$ satisfies $0 \leq \operatorname{ess} \inf \Im(\sigma) \leq$ $\operatorname{ess} \sup \Im(\sigma)<+\infty$, and $0<\operatorname{ess} \inf \Re(\sigma) \leq \operatorname{ess} \sup \Re(\sigma)<+\infty .^{2}$ Let $f \in L^{2}\left(\mathbb{R}^{\bar{d}}\right)$ with supp $f \subset B_{4} \backslash B_{1}$, and let $v \in H_{\text {loc }}^{1}\left(\mathbb{R}^{d}\right)$ be the unique outgoing solution of

$$
\operatorname{div}(A \nabla v)+k^{2} \Sigma v=f \quad \text { in } \mathbb{R}^{d}
$$

with

$$
A, \Sigma=\left\{\begin{array}{cl}
I, 1 & \text { in } \mathbb{R}^{d} \backslash B_{1}, \\
I, 1+i /(k \lambda) & \text { in } B_{1} \backslash B_{1 / 2}, \\
a, \sigma & \text { in } B_{1 / 2} .
\end{array}\right.
$$

Then for any $k_{0}>0$, there exists $C>0$ such that

\footnotetext{
${ }^{2}$ In this paper, we use the notation $\Im(\xi)$ and $\Re(\xi)$ for the imaginary part and the real part of $\xi$, respectively.
}

Copyright $@$ by SIAM. Unauthorized reproduction of this article is prohibited. 
(a) for $k>k_{0}$ and $\beta>1$

$$
\frac{1}{\beta} \int_{B_{\beta} \backslash B_{1}}\left(|\nabla v|^{2}+k^{2}|v|^{2}\right) \leq C \int_{\mathbb{R}^{d}}|f|^{2}
$$

(b) for $0<k<k_{0}$ and $\beta>1$

$$
\left\{\begin{array}{cc}
\|v\|_{L^{2}\left(B_{\beta} \backslash B_{1}\right)} \leq C \beta^{\frac{1}{2}} \max \{1, \lambda / k\}\|f\|_{L^{2}} & \text { for } d=3, \\
\|v\|_{L^{2}\left(B_{2 \beta} \backslash B_{\beta}\right)} \leq C \beta \max \{1, \lambda / k\} \frac{\left|H_{0}^{(1)}(k \beta)\right|}{\left|H_{0}^{(1)}(k)\right|}\|f\|_{L^{2}} & \text { for } d=2 .
\end{array}\right.
$$

The constant $C$ depends on $k_{0}$ but is independent of $a, \sigma, k, \beta, \lambda$, and $f$.

The following proposition, which is an immediate consequence of Lemma 1, will be used in the proofs of Theorems 1 and 2 .

Proposition 3. Let $d=2,3,0<\lambda<1$, and $k>0$. Let a be a real, symmetric matrix valued function, and let $\sigma$ be a complex function, both defined on $B_{1 / 2}$. Suppose $a$ is bounded and uniformly elliptic, and suppose $\sigma$ satisfies $0 \leq \operatorname{ess} \inf \Im(\sigma) \leq$ ess $\sup \Im(\sigma)<+\infty$, and $0<\operatorname{ess} \inf \Re(\sigma) \leq \operatorname{ess} \sup \Re(\sigma)<+\infty$. Let $V \in H^{1}\left(B_{4} \backslash \bar{B}_{1}\right)$ be such that $\Delta V+k^{2} V=0$ in $B_{4} \backslash \bar{B}_{1}, V=0$ on $\partial B_{1}$. Assume that $v \in H_{l o c}^{1}\left(\mathbb{R}^{d}\right)$ is the unique outgoing solution of the system ${ }^{3}$

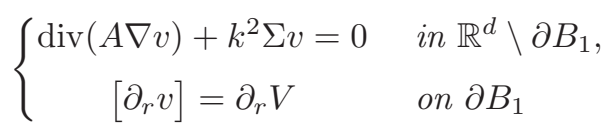

with

$$
A, \Sigma=\left\{\begin{array}{cl}
I, 1 & \text { in } \mathbb{R}^{d} \backslash B_{1}, \\
I, 1+i /(k \lambda) & \text { in } B_{1} \backslash B_{1 / 2}, \\
a, \sigma & \text { in } B_{1 / 2} .
\end{array}\right.
$$

Then for any $k_{0}>0$, there exists $C>0$ such that

(a) for $k>k_{0}$ and $\beta>1$

$$
\frac{1}{\beta} \int_{B_{2 \beta} \backslash B_{\beta}} k^{2}|v|^{2} \leq C\|V\|_{H^{1}\left(B_{4} \backslash B_{1}\right)}^{2}+\frac{2}{\beta} \int_{B_{4} \backslash B_{\beta}} k^{2}|V|^{2} ;
$$

(b) for $0<k<k_{0}$ and $\beta>1$,

$$
\|v\|_{L^{2}\left(B_{2 \beta} \backslash B_{\beta}\right)} \leq C \beta^{\frac{1}{2}} \max \{1, \lambda / k\}\|V\|_{H^{1}\left(B_{4} \backslash B_{1}\right)}
$$

for $d=3$, and

$$
\|v\|_{L^{2}\left(B_{2 \beta} \backslash B_{\beta}\right)} \leq C \beta \max \{1, \lambda / k\} \frac{\left|H_{0}^{(1)}(k \beta)\right|}{\left|H_{0}^{(1)}(k)\right|}\|V\|_{H^{1}\left(B_{4} \backslash B_{1}\right)}
$$

for $d=2$.

The constant $C$ depends on $k_{0}$ but is independent of $a, \sigma, k, \beta, \lambda$, and $V$.

\footnotetext{
${ }^{3}$ In this paper, we use the notation $[v]$ to denote $\left.v\right|_{\text {ext }}-\left.v\right|_{\text {int }}$ on $\partial D$ for any $D \subset \mathbb{R}^{d}$.
}

Copyright (c) by SIAM. Unauthorized reproduction of this article is prohibited. 
Proof of Proposition 3. Let $\phi \in C^{2}\left(\mathbb{R}^{d}\right)$ be such that $\operatorname{supp} \phi \subset B_{4}, 0 \leq \phi \leq 1$, and $\phi=1$ in $B_{3}$. Define

$$
v_{1}=\left\{\begin{array}{cl}
v-\phi V & \text { if } x \in \mathbb{R}^{d} \backslash B_{1}, \\
v & \text { if } x \in B_{1} .
\end{array}\right.
$$

Since $V=0$ on $\partial B_{1}$ and $[\langle A \nabla v, x /|x|\rangle]=\partial_{r} V$, it follows that $v_{1} \in H_{l o c}^{1}\left(\mathbb{R}^{d}\right)$ is the unique outgoing solution to the equation

$$
\operatorname{div}\left(A \nabla v_{1}\right)+k^{2} \Sigma v_{1}=f \quad \text { in } \mathbb{R}^{d},
$$

where

$$
f=\left\{\begin{array}{cl}
-\left(\Delta V+k^{2} V\right) \phi-2 \nabla V \nabla \phi-V \Delta \phi & \text { in } \mathbb{R}^{d} \backslash B_{1}, \\
0 & \text { in } B_{1} .
\end{array}\right.
$$

It is clear that supp $f \subset B_{4} \backslash B_{1}$, and since $\Delta V+k^{2} V=0$ in $B_{4} \backslash B_{1}$,

$$
\|f\|_{L^{2}} \leq C\|V\|_{H^{1}\left(B_{4} \backslash B_{1}\right)} .
$$

The estimates of this proposition now follow from Lemma 1 and the fact that

$$
\frac{\beta\left|H_{0}^{(1)}(k \beta)\right|}{\left|H_{0}^{(1)}(k)\right|} \geq c>0 \text { for } 0<k<k_{0} \text { and } \beta>1 .
$$

2.2. New estimates for Helmholtz problems in the low frequency regime. In this section we improve the low frequency results from the previous section, under the additional assumption that $a$ and $\sigma$ are in finite ranges. The notion of G-convergence plays a role in the proof of these improved results. We therefore first recall the definition of $G$-convergence and state one of the fundamental results associated with this notion. We emphasize that if we considered cloaking for fixed $a$ and $\sigma$, then our approach would work without the use of $G$-convergence.

2.2.1. $G$-convergence. We recall here the definition of a particular version of $G$-convergence (also frequently referred to as $H$-convergence) and state a central theorem involving this notion.

Suppose $0<\alpha<\beta<\infty$ and let $\Omega$ be a connected, bounded, smooth open subset of $\mathbb{R}^{d}$. $M(\alpha, \beta, \Omega)$ denotes the set of real symmetric matrix valued functions $a$ defined on $\Omega$ such that

$$
\alpha|\xi|^{2} \leq\langle a \xi, \xi\rangle \leq \beta|\xi|^{2}
$$

and $H_{\sharp}^{1}(\Omega)$ denotes the Sobolev space

$$
H_{\sharp}^{1}(\Omega)=\left\{\psi \in H^{1}(\Omega) ; \int_{\Omega} \psi=0\right\} .
$$

Definition 1. A sequence of matrices $\left(a_{n}\right)$ in $M(\alpha, \beta, \Omega) G$-converges to a matrix $a \in M\left(\alpha^{\prime}, \beta^{\prime}, \Omega\right)$ iff for all $g \in\left[H^{1}(\Omega)\right]^{*}$ (the dual of $\left.H^{1}(\Omega)\right)$ with $\langle g, 1\rangle_{\left[H^{1}\right]^{*}, H^{1}}=$ 0 , the solution $u_{n} \in H_{\sharp}^{1}(\Omega)$ of the equation

$$
\operatorname{div}\left(a_{n} \nabla u_{n}\right)=g, \quad \text { i.e., } \int_{\Omega} a_{n} \nabla u_{n} \nabla \psi=\langle g, \psi\rangle_{\left[H^{1}\right]^{*}, H^{1}} \quad \forall \psi \in H_{\sharp}^{1}(\Omega),
$$

Copyright $@$ by SIAM. Unauthorized reproduction of this article is prohibited. 
has the property that

$$
u_{n} \rightarrow u \text { weakly in } H^{1}(\Omega) \quad \text { and } \quad a_{n} \nabla u_{n} \rightarrow a \nabla u \text { weakly in } L^{2}(\Omega),
$$

where $u \in H_{\sharp}^{1}(\Omega)$ is the unique solution of the problem

$$
\operatorname{div}(a \nabla u)=g, \quad \text { i.e., } \int_{\Omega} a \nabla u \nabla \psi=\langle g, \psi\rangle_{\left[H^{1}\right]^{*}, H^{1}} \quad \forall \psi \in H_{\sharp}^{1}(\Omega) .
$$

Concerning $G$-convergence, one has the following.

Proposition 4. Let $0<\alpha<\beta<+\infty$, let $\Omega$ be a connected, bounded, and smooth subset of $\mathbb{R}^{d}$, and suppose $\left(a_{n}\right) \subset M(\alpha, \beta, \Omega)$; then

(i) there exist a subsequence $\left(a_{n_{k}}\right)$ of $\left(a_{n}\right)$ and $a \in M\left(\alpha^{\prime}, \beta^{\prime}, \Omega\right)$ for some $0<$ $\alpha^{\prime}<\beta^{\prime}<+\infty$ such that $\left(a_{n_{k}}\right) G$-converges to a;

(ii) suppose $\left(a_{n}\right),\left(b_{n}\right) \subset M(\alpha, \beta, \Omega)$ with $a_{n}=b_{n}$ on an open subset $\Omega^{\prime}$ of $\Omega$, and suppose $a_{n} G$-converges to $a \in M\left(\alpha^{\prime}, \beta^{\prime}, \Omega\right), b_{n} G$-converges to $b \in$ $M\left(\alpha^{\prime}, \beta^{\prime}, \Omega\right)$ for some $0<\alpha^{\prime}<\beta^{\prime}<+\infty$. Then $a=b$ on $\Omega^{\prime}$.

Proof of Proposition 4. The result analogous to Proposition 4 for the zero-Dirichlet boundary condition is well-known; see, e.g., [7] and [14]. The proof of Proposition 4 follows by a slight variation of the proof for the zero-Dirichlet boundary condition. The details are left to the reader.

2.2.2. New estimates in the $3 \mathrm{~d}$ low frequency regime. This section is devoted to new estimates for the Helmholtz equation in the $3 \mathrm{~d}$ low frequency regime. The main result is the following proposition.

Proposition 5. Let a be a real, symmetric matrix valued function, and let $\sigma$ be a complex function, both defined on $B_{1 / 2}$. Assume that

$$
\frac{1}{\Lambda}|\xi|^{2} \leq\langle a \xi, \xi\rangle \leq \Lambda|\xi|^{2}, \quad \frac{1}{\Lambda} \leq \Re(\sigma) \leq \Lambda, \quad \text { and } \quad 0 \leq \Im(\sigma) \leq \Lambda
$$

for some positive constant $\Lambda$. Given $0<k<1$ and $0<\varepsilon<1$, let $V_{\varepsilon} \in H^{1}\left(B_{4} \backslash \bar{B}_{1}\right)$ be such that $\Delta V_{\varepsilon}+\varepsilon^{2} k^{2} V_{\varepsilon}=0$ in $B_{4} \backslash B_{1}, V_{\varepsilon}=0$ on $\partial B_{1}$, and let $v_{\varepsilon} \in H_{\text {loc }}^{1}\left(\mathbb{R}^{3}\right)$ be the unique outgoing solution of the system

$$
\begin{cases}\operatorname{div}\left(A_{\varepsilon} \nabla v_{\varepsilon}\right)+\varepsilon^{2} k^{2} \Sigma_{\varepsilon} v_{\varepsilon}=0 & \text { in } \mathbb{R}^{3} \backslash \partial B_{1}, \\ {\left[\partial_{r} v_{\varepsilon}\right]=\partial_{r} V_{\varepsilon}} & \text { on } \partial B_{1}\end{cases}
$$

with

$$
A_{\varepsilon}, \Sigma_{\varepsilon}=\left\{\begin{array}{cl}
I, 1 & \text { in } \mathbb{R}^{3} \backslash B_{1}, \\
I, 1+i /\left(\varepsilon^{2+\gamma} k\right) & \text { in } B_{1} \backslash B_{1 / 2}, \\
\frac{1}{\varepsilon} a, \frac{1}{\varepsilon^{3}} \sigma & \text { in } B_{1 / 2}
\end{array}\right.
$$

for some constant $\gamma \geq 0$. Then there exist two positive constants $c$ and $C$ such that $k<c \min \left\{\varepsilon^{1 / 2}, \varepsilon^{\gamma}\right\}$ implies

$$
\left\|v_{\mathcal{E}}\right\|_{L^{2}\left(B_{2 \beta} \backslash B_{\beta}\right)} \leq C \beta^{\frac{1}{2}}\left\|V_{\varepsilon}\right\|_{H^{1}\left(B_{4} \backslash B_{1}\right)} \quad \forall \beta>1 .
$$

The constants $c$ and $C$ depend on the "range" constant $\Lambda$ and on $\gamma$ but are independent of $V_{\varepsilon}, k, \varepsilon, \beta, a$, and $\sigma$. 
Proposition 5 is a consequence of Lemma 2 below. The proof of Proposition 5 is similar to the one of Proposition 3, where instead of using Lemma 1, one uses Lemma 2. The details are left to the reader.

LEMMA 2. Let a be a real, symmetric matrix valued function, and let $\sigma$ be a complex function, both defined on $B_{1 / 2}$. Assume that

$$
\frac{1}{\Lambda}|\xi|^{2} \leq\langle a \xi, \xi\rangle \leq \Lambda|\xi|^{2}, \quad \frac{1}{\Lambda} \leq \Re(\sigma) \leq \Lambda, \quad \text { and } \quad 0 \leq \Im(\sigma) \leq \Lambda
$$

for some positive constant $\Lambda$. Given $0<k<1,0<\varepsilon<1$, and $f \in L^{2}\left(\mathbb{R}^{3}\right)$ with $\operatorname{supp} f \subset B_{4} \backslash B_{1}$, let $v_{\varepsilon} \in H_{\text {loc }}^{1}\left(\mathbb{R}^{3}\right)$ be the unique outgoing solution of

$$
\operatorname{div}\left(A_{\varepsilon} \nabla v_{\varepsilon}\right)+\varepsilon^{2} k^{2} \Sigma_{\varepsilon} v_{\varepsilon}=f \quad \text { in } \mathbb{R}^{3}
$$

with

$$
A_{\varepsilon}, \Sigma_{\varepsilon}=\left\{\begin{array}{cl}
I, 1 & \text { in } \mathbb{R}^{3} \backslash B_{1}, \\
I, 1+i /\left(\varepsilon^{2+\gamma} k\right) & \text { in } B_{1} \backslash B_{1 / 2}, \\
\frac{1}{\varepsilon} a, \frac{1}{\varepsilon^{3}} \sigma & \text { in } B_{1 / 2}
\end{array}\right.
$$

for some constant $\gamma \geq 0$. Then there exist two positive constants $c$ and $C$ such that $k<c \min \left\{\varepsilon^{1 / 2}, \varepsilon^{\gamma}\right\}$ implies

$$
\left\|v_{\varepsilon}\right\|_{L^{2}\left(B_{5}\right)} \leq C\|f\|_{L^{2}}
$$

and

$$
\left\|v_{\varepsilon}\right\|_{L^{2}\left(B_{2 \beta} \backslash B_{\beta}\right)} \leq C \beta^{1 / 2}\|f\|_{L^{2}} .
$$

The constants $c$ and $C$ depend on the "range" constant $\Lambda$ and $\gamma$ but are independent of $f, k, \varepsilon, \beta, a$, and $\sigma$.

Proof of Lemma 2. We only establish (2.8). The estimate (2.9) for $\beta>4.5$ follows immediately from (2.8) and Proposition 2, since

$$
\left\|v_{\varepsilon}\right\|_{H^{1 / 2}\left(\partial B_{4.5}\right)} \leq C\left\|v_{\varepsilon}\right\|_{H^{1}\left(B_{4.8} \backslash B_{4.2}\right)} \leq C\left\|v_{\varepsilon}\right\|_{L^{2}\left(B_{5} \backslash B_{4}\right)},
$$

by standard regularity theory for elliptic equations. The extension of (2.9) to all $\beta>1$ is a simple consequence of $(2.8)$.

The proof of (2.8) uses ideas from [16, Lemma 3] and the theory of $G$-convergence (Proposition 4) and proceeds by contradiction. Suppose (2.8) is not true; then there exist $k_{n} \rightarrow 0,0<\varepsilon_{n}<1, a_{n}, \sigma_{n}$ that satisfy (2.6), and $\left\{f_{n}\right\}$ with supp $f_{n} \subset B_{4} \backslash B_{1}$, such that

$$
\lim _{n \rightarrow \infty} k_{n} / \min \left\{\varepsilon_{n}^{1 / 2}, \varepsilon_{n}^{\gamma}\right\}=0, \quad\left\|f_{n}\right\|_{L^{2}} \rightarrow 0, \quad \text { and } \quad\left\|v_{n}\right\|_{L^{2}\left(B_{5}\right)}=1 .
$$

Here $v_{n} \in H_{\text {loc }}^{1}\left(\mathbb{R}^{3}\right)$ is the unique outgoing solution to the equation

$$
\operatorname{div}\left(A_{n} \nabla v_{n}\right)+\varepsilon_{n}^{2} k_{n}^{2} \Sigma_{n} v_{n}=f_{n} \quad \text { in } \mathbb{R}^{3},
$$

where $A_{n}$ and $\Sigma_{n}$ are defined similarly to $A_{\varepsilon}$ and $\Sigma_{\varepsilon}$ of $(2.7)$ with $a, \sigma$, $\varepsilon$, and $k$ replaced by $a_{n}, \sigma_{n}, \varepsilon_{n}$, and $k_{n}$, respectively. Applying Proposition 2 with $D=B_{9 / 2}$, elliptic estimates, and (2.10), we obtain

$$
\left\|v_{n}\right\|_{H^{1}(K)} \leq C_{K}
$$

Copyright (c) by SIAM. Unauthorized reproduction of this article is prohibited. 
for any compact subset $K$ of $\mathbb{R}^{3} \backslash B_{9 / 2}$. Multiplication of (2.11) by $\bar{v}_{n}$ (the conjugate of $v_{n}$ ) and integration of the obtained expression on $B_{5}$ yields

$$
\begin{aligned}
\int_{B_{5} \backslash B_{1 / 2}}\left|\nabla v_{n}\right|^{2}+\frac{1}{\varepsilon_{n}} \int_{B_{1 / 2}}\left\langle a_{n} \nabla v_{n}, \nabla v_{n}\right\rangle-\varepsilon_{n}^{2} k_{n}^{2} \int_{B_{5} \backslash B_{1 / 2}}\left|v_{n}\right|^{2} \\
-\frac{i k_{n}}{\varepsilon_{n}^{\gamma}} \int_{B_{1} \backslash B_{1 / 2}}\left|v_{n}\right|^{2}-\frac{k_{n}^{2}}{\varepsilon_{n}} \int_{B_{1 / 2}} \sigma_{n}\left|v_{n}\right|^{2}=-\int_{B_{5}} f_{n} \bar{v}_{n}+\int_{\partial B_{5}} \partial_{r} v_{n} \bar{v}_{n} .
\end{aligned}
$$

From (2.10), (2.12), (2.13), and the fact that $a_{n}$ and $\sigma_{n}$ satisfy (2.6), we have

$$
\left\|v_{n}\right\|_{H^{1}\left(B_{5}\right)} \leq C
$$

where $C$ depends on $\Lambda$. Hence it follows from (2.12) that

$$
\left\|v_{n}\right\|_{H^{1}(K)} \leq C_{K} \quad \forall K \subset \subset \mathbb{R}^{3} .
$$

Set

$$
v_{1, n}=f_{B_{1 / 2}} v_{n}
$$

and define $v_{2, n}, w_{n}$ on $B_{1 / 2}$ as follows:

$$
v_{2, n}=v_{n}-v_{1, n} \quad \text { and } \quad w_{n}=\frac{1}{\varepsilon_{n}} v_{2, n} .
$$

From the equation for $v_{n}$, we have

$$
\operatorname{div}\left(a_{n} \nabla v_{n}\right)+k_{n}^{2} \sigma_{n} v_{n}=0 \text { in } B_{1 / 2} \quad \text { and }\left.\quad a_{n} \nabla v_{n} \cdot \frac{x}{|x|}\right|_{\text {int }}=\left.\varepsilon_{n} \partial_{r} v_{n}\right|_{\text {ext }} \text { on } \partial B_{1 / 2} \text {. }
$$

This implies

$$
\operatorname{div}\left(a_{n} \nabla w_{n}\right)=-\frac{k_{n}^{2} \sigma_{n}}{\varepsilon_{n}} v_{n} \text { in } B_{1 / 2}, \quad a_{n} \nabla w_{n} \cdot \frac{x}{|x|}=\left.\partial_{r} v_{n}\right|_{\text {ext }} \text { on } \partial B_{1 / 2},
$$

and $\int_{B_{1 / 2}} w_{n}=0$. Since $\left(k_{n}^{2} \sigma_{n} / \varepsilon_{n}\right) v_{n} \rightarrow 0$ in $L^{2}\left(B_{1 / 2}\right)$, it follows from (2.14) that $w_{n}$ is bounded in $H^{1}\left(B_{1 / 2}\right)$. From (2.14) and (2.16), it is clear that

$$
\left.a_{n} \nabla v_{n} \cdot \frac{x}{|x|}\right|_{\text {int }} \rightarrow 0 \text { in } H^{-1 / 2}\left(\partial B_{1 / 2}\right),
$$

and so

$$
\int_{B_{1 / 2}}\left\langle a_{n} \nabla v_{n}, \nabla v_{n}\right\rangle=k_{n}^{2} \int_{B_{1 / 2}} \sigma_{n}\left|v_{n}\right|^{2}+\left.\int_{\partial B_{1 / 2}} a_{n} \nabla v_{n} \cdot \frac{x}{|x|}\right|_{\text {int }} \bar{v}_{n} \rightarrow 0 .
$$

Due to (2.6), (2.14), and (2.19), we may (after extraction of a subsequence) assume that $\left(v_{n}\right)$ converges to a constant in $H^{1}\left(B_{1 / 2}\right)$. On the other hand, from (2.14) and the fact that $\Delta v_{n}+\varepsilon_{n}^{2} k_{n}^{2} v_{n}=0$ for $|x|>4$, we may (after extraction of a subsequence and a diagonalization argument) assume that $\left(v_{n}\right)$ converges in $H^{1}(K)$ for any $K \subset \subset \mathbb{R}^{3} \backslash B_{9 / 2}$. Hence $\left(v_{n}\right)$ converges in $H^{1 / 2}\left(\partial B_{5}\right)$ and $H^{1 / 2}\left(\partial B_{1 / 2}\right)$.

Copyright (c) by SIAM. Unauthorized reproduction of this article is prohibited. 
Using the equation for $v_{n}$ in $B_{5} \backslash \bar{B}_{1 / 2}$ and the theory of elliptic equations, we obtain that $\left(v_{n}\right)$ converges in $H^{1}\left(B_{5} \backslash \bar{B}_{1 / 2}\right)$. Here we also used $(2.14)$ and the fact that $k_{n} / \varepsilon_{n}^{\gamma} \rightarrow 0$. In summary, we have that $\left(v_{n}\right)$ converges in $H_{\text {loc }}^{1}\left(\mathbb{R}^{3}\right)$. Let $v$ be the limit of $v_{n}$ in $H_{l o c}^{1}\left(\mathbb{R}^{3}\right)$. By Proposition 4, we may (after extraction of a subsequence) assume that $\left(a_{n}\right) G$-converges to $a$ and that $\left(w_{n}\right)$ converges weakly to $w$ in $H^{1}\left(B_{1 / 2}\right)$. It now follows from (2.16) and (2.17) that

$$
\left\{\begin{array}{c}
\operatorname{div}(a \nabla w)=0 \text { in } B_{1 / 2} \quad \operatorname{div}(a \nabla v)=0 \text { in } B_{1 / 2}, \\
\left\langle a \nabla w, \frac{x}{|x|}\right\rangle=\left.\partial_{r} v\right|_{\text {ext }} \text { on } \partial B_{1 / 2} \quad\left\langle\left. a \nabla v\right|_{\text {int }}, \frac{x}{|x|}\right\rangle=0 \text { on } \partial B_{1 / 2} .
\end{array}\right.
$$

This is consistent with the fact that $v$ is constant on $B_{1 / 2}$. It is not difficult to see that $\Delta v=0$ in $\mathbb{R}^{3} \backslash B_{1 / 2}$ and that $v \in W^{1}\left(\mathbb{R}^{3}\right)$ (by Proposition 2). Since $\int_{B_{1 / 2}} w_{n}=$ 0 , it follows that $\int_{B_{1 / 2}} w=0$. Lemma 3 (stated below) now implies that $v=0$ and $w=0$. However, this contradicts the fact that $\|v\|_{L^{2}\left(B_{5}\right)}=\lim _{n \rightarrow \infty}\left\|v_{n}\right\|_{L^{2}\left(B_{5}\right)}$ $=1$.

In the proof of Lemma 2 we used the following result, which was established in [16, Lemma 2].

Lemma 3. Let a be a real, symmetric, positive definite matrix valued function defined on a connected bounded smooth domain $D$ of $\mathbb{R}^{3}$. There exists no nonzero solution $(v, w)$ in $W^{1}\left(\mathbb{R}^{3}\right) \times H_{\sharp}^{1}(D)$ of the system

$$
\left\{\begin{array} { l l } 
{ \Delta v = 0 } & { \text { in } \mathbb { R } ^ { 3 } \backslash \overline { D } , } \\
{ \operatorname { d i v } ( a \nabla v ) = 0 } & { \text { in } D , } \\
{ \operatorname { d i v } ( a \nabla w ) = 0 } & { \text { in } D , }
\end{array} \quad \text { and } \left\{\begin{array}{ll}
\left.a \nabla v \cdot \nu\right|_{\text {int }}=0 & \text { on } \partial D, \\
\left.\frac{\partial v}{\partial \nu}\right|_{\text {ext }}-a \nabla w \cdot \nu=0 & \text { on } \partial D .
\end{array}\right.\right.
$$

Here $\nu$ denotes the outward normal unit vector on $\partial D$.

The proof of Lemma 3 goes as follows.

Proof. Since $v \in W^{1}\left(\mathbb{R}^{3} \backslash \bar{D}\right)$, it follows from (2.20) that

$$
\int_{\mathbb{R}^{3} \backslash D}|\nabla v|^{2}=-\left.\int_{\partial D} \frac{\partial v}{\partial \nu}\right|_{\text {ext }} \bar{v}=-\int_{\partial D}(a \nabla w \cdot \nu) \bar{v} .
$$

We also deduce from (2.20) that

$$
\int_{\partial D}(a \nabla w \cdot \nu) \bar{v}=\int_{D} a \nabla w \nabla \bar{v}=0 .
$$

For the latter, we have used that $v$ is constant in $D$. A combination of (2.21) and (2.22) yields $v=0$ in $\mathbb{R}^{3} \backslash D$. It follows from the continuity of $v$ across $\partial D$ that

$$
v=0 \quad \text { in } \mathbb{R}^{3} \quad \text { and } \quad w=0 \text { in } D .
$$

2.2.3. New estimates in the $2 d$ low frequency regime. In this section, we establish new estimates for the Helmholtz equation in the $2 \mathrm{~d}$ low frequency regime. These estimates will play an important role for the analysis of cloaking for the full wave equation in $2 \mathrm{~d}$. The analogue of Proposition 5 is as follows.

Proposition 6. Let a be a real, symmetric matrix valued function, and let $\sigma$ be a complex function, both defined on $B_{1 / 2}$. Assume that

$$
\frac{1}{\Lambda}|\xi|^{2} \leq\langle a \xi, \xi\rangle \leq \Lambda|\xi|^{2}, \quad \frac{1}{\Lambda} \leq \Re(\sigma) \leq \Lambda, \quad \text { and } \quad 0 \leq \Im(\sigma) \leq \Lambda
$$

Copyright $@$ by SIAM. Unauthorized reproduction of this article is prohibited. 
for some positive constant $\Lambda$. Given $0<k<1$, $0<\lambda<1$, $0<\varepsilon<1$, let $V_{\varepsilon} \in$ $H^{1}\left(B_{4} \backslash \bar{B}_{1}\right)$ be such that $\Delta V_{\varepsilon}+\varepsilon^{2} k^{2} V_{\varepsilon}=0$ in $B_{4} \backslash B_{1}, V_{\varepsilon}=0$ on $\partial B_{1}$, and let $v_{\varepsilon} \in H_{\text {loc }}^{1}\left(\mathbb{R}^{2}\right)$ be the unique outgoing solution of the system

$$
\begin{cases}\operatorname{div}\left(A \nabla v_{\varepsilon}\right)+\varepsilon^{2} k^{2} \Sigma_{\varepsilon} v_{\varepsilon}=0 & \text { in } \mathbb{R}^{2} \backslash \partial B_{1}, \\ {\left[\partial_{r} v_{\varepsilon}\right]=\partial_{r} V_{\varepsilon}} & \text { on } \partial B_{1}\end{cases}
$$

with

$$
A, \Sigma_{\varepsilon}=\left\{\begin{array}{cl}
I, 1 & \text { in } \mathbb{R}^{2} \backslash B_{1}, \\
I, 1+i /(\varepsilon k \lambda) & \text { in } B_{1} \backslash B_{1 / 2}, \\
a, \frac{1}{\varepsilon^{2}} \sigma & \text { in } B_{1 / 2} .
\end{array}\right.
$$

There exist two positive constants $c$ and $C$, depending only on $\Lambda$, such that if $\varepsilon k<c$, $\varepsilon k|\ln (\varepsilon k)|^{2}<c \lambda$, and $k^{2}|\ln (\varepsilon k)|^{2}<c$, then

$$
\left\|v_{\varepsilon}\right\|_{L^{2}\left(B_{5}\right)} \leq C|\ln (\varepsilon k)|^{2}\left\|V_{\varepsilon}\right\|_{H^{1}\left(B_{4} \backslash B_{1}\right)}
$$

and

$$
\left\|v_{\varepsilon}\right\|_{L^{2}\left(B_{2 \beta} \backslash B_{\beta}\right)} \leq C \beta\left|H_{0}^{(1)}(\varepsilon k \beta)\|\ln (\varepsilon k) \mid\| V_{\varepsilon} \|_{H^{1}\left(B_{4} \backslash B_{1}\right)} \quad \forall \beta>1 .\right.
$$

The constants $c$ and $C$ depend on the "range" constant $\Lambda$ but are independent of $V_{\varepsilon}$, $k, \varepsilon, \lambda, a, \sigma$, and $\beta$.

Proposition 6 is a consequence of Lemma 5 (and Lemma 4) below. The proof of Proposition 6 is similar to the one of Proposition 5, where instead of Lemma 2, we use Lemma 5. The details of this proof are left for the reader.

The following result plays an important role in $2 \mathrm{~d}$ low frequency regime.

Lemma 4. Let a be a real, symmetric matrix valued function defined on $B_{1 / 2}$. Assume that

$$
\frac{1}{\Lambda}|\xi|^{2} \leq\langle a \xi, \xi\rangle \leq \Lambda|\xi|^{2}
$$

for some positive constant $\Lambda$. Given $f \in L^{2}\left(\mathbb{R}^{2}\right)$ with $\operatorname{supp} f \subset B_{4} \backslash B_{1}$, let $v_{\varepsilon} \in$ $H_{\text {loc }}^{1}\left(\mathbb{R}^{2}\right)$ be the outgoing solution of the Helmholtz equation

$$
\operatorname{div}\left(A \nabla v_{\varepsilon}\right)+\varepsilon^{2} v_{\varepsilon}=f
$$

with $A=I$ in $\mathbb{R}^{2} \backslash B_{1 / 2}$ and $A=a$ in $B_{1 / 2}$. There exist constants $c$ and $C$ depending only on $\Lambda$ such that $0<\varepsilon<c$ implies

$$
\left\|v_{\varepsilon}\right\|_{L^{2}\left(B_{5}\right)} \leq C|\ln \varepsilon|^{2}\|f\|_{L^{2}} .
$$

Remark 5. Lemma 4 is obvious if $a=I$; the proof follows immediately from the behavior of the fundamental solution of the Helmholtz equation in $2 \mathrm{~d}$. In fact, in this case we have a better estimate:

$$
\left\|v_{\varepsilon}\right\|_{L^{2}\left(B_{5}\right)} \leq C|\ln \varepsilon|\|f\|_{L^{2}}
$$

We believe that this estimate also holds in the setting of Lemma 4; however, we do not know how to prove it. The weaker estimate in Lemma 4 is sufficient to obtain our desired cloaking estimate in the $2 \mathrm{~d}$ case.

Copyright $@$ by SIAM. Unauthorized reproduction of this article is prohibited. 
We are ready to give the following.

Proof of Lemma 4. We proceed by contradiction. Suppose there exist a sequence $\varepsilon_{n} \rightarrow 0$, a sequence $\left(f_{n}\right) \subset L^{2}\left(\mathbb{R}^{2}\right)$, and a sequence $\left(a_{n}\right)$ of symmetric matrices such that $\operatorname{supp} f_{n} \subset B_{4} \backslash B_{1},(1 / \Lambda)|\xi|^{2} \leq\left\langle a_{n} \xi, \xi\right\rangle \leq \Lambda|\xi|^{2}$,

$$
\lim _{n \rightarrow \infty}\left|\ln \varepsilon_{n}\right|^{2}\left\|f_{n}\right\|_{L^{2}}=0, \quad \text { and } \quad\left\|v_{n}\right\|_{L^{2}\left(B_{5}\right)}=1 .
$$

Here $v_{n} \in H_{l o c}^{1}\left(\mathbb{R}^{2}\right)$ is the unique outgoing solution to the equation

$$
\operatorname{div}\left(A_{n} \nabla v_{n}\right)+\varepsilon_{n}^{2} v_{n}=f_{n}
$$

with $A_{n}=I$ for $x \in \mathbb{R}^{2} \backslash B_{1 / 2}$ and $A_{n}=a_{n}$ otherwise. Applying Proposition 2 with $D=B_{9 / 2}$, using elliptic estimates, and (2.24), we obtain

$$
\left\|v_{n}\right\|_{H^{1}(K)} \leq C_{K}
$$

for any compact subset $K$ of $\mathbb{R}^{2} \backslash B_{9 / 2}$. Multiplication of (2.25) by $\bar{v}_{n}$ and integration of the obtained expression on $B_{5}$ yields

$$
\int_{B_{5}}\left\langle A_{n} \nabla v_{n}, \nabla v_{n}\right\rangle-\varepsilon_{n}^{2} \int_{B_{5}}\left|v_{n}\right|^{2}=-\int_{B_{5}} f_{n} \bar{v}_{n}+\int_{\partial B_{5}} \frac{\partial v_{n}}{\partial r} \bar{v}_{n} .
$$

It follows from (2.24), (2.26), and (2.27) that

$$
\left\|v_{n}\right\|_{H^{1}\left(B_{5}\right)} \leq C
$$

where $C$ depends on $\Lambda$. A combination of (2.26) and (2.28) yields

$$
\left\|v_{n}\right\|_{H^{1}\left(B_{R}\right)} \leq C_{R} \quad \forall R>0 .
$$

From the second part of Proposition 2 and Proposition 4, it now follows that there exists a symmetric matrix $a \in M\left(\alpha, \beta, B_{1 / 2}\right)$ for some $0<\alpha<\beta<+\infty$, such that (after extraction of a subsequence) $v_{n} \rightarrow v$ in $L_{l o c}^{2}\left(\mathbb{R}^{2}\right)$, where $v \in W^{1}\left(\mathbb{R}^{2}\right)$ is a solution to the equation

$$
\operatorname{div}(A \nabla v)=0 .
$$

Here $A=I$ for $x \in \mathbb{R}^{2} \backslash B_{1 / 2}$ and $A=a$ otherwise. It is clear that $v=\alpha$ for some (complex) constant $\alpha$. Since $\Delta v_{n}+\varepsilon_{n}^{2} v_{n}=0$ in $\mathbb{R}^{2} \backslash B_{4}$, and $v_{n}$ satisfies the outgoing radiation condition, $v_{n}$ can be represented as

$$
v_{n}(x)=\sum_{l=-\infty}^{\infty} a_{l, n} H_{l}^{(1)}\left(\varepsilon_{n}|x|\right) e^{i l \theta}, \quad|x|>4,
$$

where $H_{l}^{(1)}$ is the Hankel function of the first kind of order $l$. This implies

$$
v_{n}=v_{0, n}+v_{1, n}, \quad|x|>4,
$$

where

$$
v_{0, n}=a_{0, n} H_{0}^{(1)}\left(\varepsilon_{n}|x|\right) \quad \text { and } \quad v_{1, n}=\sum_{l \neq 0} a_{l, n} H_{l}^{(1)}\left(\varepsilon_{n}|x|\right) e^{i l \theta}, \quad|x|>4 .
$$

Copyright $@$ by SIAM. Unauthorized reproduction of this article is prohibited. 
We recall that (see, e.g., [21])

$$
\begin{gathered}
\lim _{r \rightarrow 0} \frac{1}{|\ln r|} H_{0}^{(1)}(r)=\frac{2}{i \pi}, \quad\left|H_{0}^{(1)}\right| \searrow \text { on } \mathbb{R}_{+}, \quad \lim _{r \rightarrow 0} r \frac{d H_{0}^{(1)}(r)}{d r}=-\frac{2}{i \pi}, \\
\lim _{r \rightarrow \infty} \sqrt{\frac{\pi r}{2}} e^{-i(r-\pi / 4)} H_{0}^{(1)}(r)=1,
\end{gathered}
$$

and

$$
\int_{\partial B_{t}}\left|H_{l}^{(1)}\right|^{2} \leq \int_{\partial B_{s}}\left|H_{l}^{(1)}\right|^{2}, \quad 0<s<t
$$

for all $l \neq 0$. By orthogonality, it is clear that for any $R>4$,

$$
\left\|v_{0, n}\right\|_{H^{1}\left(B_{R} \backslash B_{4}\right)}+\left\|v_{1, n}\right\|_{H^{1}\left(B_{R} \backslash B_{4}\right)} \leq C\left\|v_{n}\right\|_{H^{1}\left(B_{R} \backslash B_{4}\right)} .
$$

Hence, after extraction of a subsequence, we may assume that $v_{0, n} \rightarrow \alpha_{0}$ in $L_{\text {loc }}^{2}\left(\mathbb{R}^{2}\right)$ and $v_{1, n} \rightarrow v_{1}$ in $L_{l o c}^{2}\left(\mathbb{R}^{2}\right)$ for some (complex) constant $\alpha_{0}$ and some $v_{1} \in L_{\text {loc }}^{2}\left(\mathbb{R}^{2}\right)$. Therefore,

$$
\alpha=v=\alpha_{0}+v_{1}, \quad|x|>4 .
$$

This implies that $v_{1}$ is constant on $\{|x|>4\}$. From (2.33) we deduce that

$$
\int_{B_{t+1} \backslash B_{t}}\left|v_{1}\right|^{2} \leq \int_{B_{s+1} \backslash B_{s}}\left|v_{1}\right|^{2}, \quad 4<s<t,
$$

and so $v_{1}$ must be equal to 0 . Thus $v_{0, n} \rightarrow v=\alpha$ in $L_{l o c}^{2}\left(\mathbb{R}^{2} \backslash B_{4}\right)$. From (2.30) and (2.31), we now have

$$
\lim _{n \rightarrow \infty}\left|a_{0, n}\right|\left|\ln \varepsilon_{n}\right|=\frac{\pi}{2}|\alpha| .
$$

On the other hand, the outgoing radiation condition implies

$$
\lim _{R \rightarrow \infty} \varepsilon_{n} \int_{\partial B_{R}}\left|v_{n}\right|^{2} \leq \int_{\mathbb{R}^{2}}\left|f_{n}\right|\left|v_{n}\right|
$$

By orthogonality and (2.32),

$$
\lim _{R \rightarrow \infty} \varepsilon_{n} \int_{\partial B_{R}}\left|v_{n}\right|^{2} \geq \lim _{R \rightarrow \infty} \varepsilon_{n} \int_{\partial B_{R}}\left|v_{0, n}\right|^{2}=\lim _{R \rightarrow \infty} 2 \pi \varepsilon_{n} R\left|a_{0, n} H_{0}^{(1)}\left(\varepsilon_{n} R\right)\right|^{2}=4\left|a_{0, n}\right|^{2} .
$$

Since $\left\|v_{n}\right\|_{L^{2}\left(B_{5}\right)}=1$ and $\operatorname{supp} f \subset B_{4}$,

$$
\int_{\mathbb{R}^{2}}\left|f_{n}\left\|v_{n} \mid \leq C\right\| f_{n} \|_{L^{2}}\right.
$$

A combination of (2.24), (2.34), (2.35), (2.36), and (2.37) yields that $v=\alpha=0$, which in turn contradicts the fact that $\left\|v_{n}\right\|_{L^{2}\left(B_{5}\right)}=1$. This completes the proof of (2.23).

Based on Lemma 4 we now state and prove the $2 \mathrm{~d}$ analogue of Lemma 2.

Lemma 5. Assume that

$$
\frac{1}{\Lambda}|\xi|^{2} \leq\langle a \xi, \xi\rangle \leq \Lambda|\xi|^{2}, \quad \frac{1}{\Lambda} \leq \Re(\sigma) \leq \Lambda, \quad \text { and } \quad 0 \leq \Im(\sigma) \leq \Lambda
$$

Copyright $@$ by SIAM. Unauthorized reproduction of this article is prohibited. 
for some positive constant $\Lambda$. Let $f \in L^{2}\left(\mathbb{R}^{2}\right)$ with $\operatorname{supp} f \subset B_{4} \backslash B_{1}$, and let $v_{\varepsilon} \in$ $H_{\text {loc }}^{1}\left(\mathbb{R}^{2}\right)$ be the unique outgoing solution of

$$
\operatorname{div}\left(A \nabla v_{\varepsilon}\right)+\varepsilon^{2} k^{2} \Sigma_{\varepsilon} v_{\varepsilon}=f \quad \text { in } \mathbb{R}^{2},
$$

where $A$ and $\Sigma_{\varepsilon}$ are as in Proposition 6 . There exist two positive constants $c$ and $C$, depending only on $\Lambda$, such that if $0<\varepsilon k<c, \varepsilon k|\ln (\varepsilon k)|^{2}<c \lambda$, and $k^{2}|\ln (\varepsilon k)|^{2}<c$, then

$$
\left\|v_{\varepsilon}\right\|_{L^{2}\left(B_{5}\right)} \leq C|\ln (\varepsilon k)|^{2}\|f\|_{L^{2}}
$$

and

$$
\left\|v_{\varepsilon}\right\|_{L^{2}\left(B_{2 \beta} \backslash B_{\beta}\right)} \leq C \beta\left|H_{0}^{(1)}(\varepsilon k \beta)\|\ln (\varepsilon k) \mid\| V_{\varepsilon} \|_{H^{1}\left(B_{4} \backslash B_{1}\right)} \quad \forall \beta>1 .\right.
$$

The constants $c$ and $C$ depend on the "range" constant $\Lambda$ but are otherwise independent of $a, \sigma, f, k, \varepsilon, \lambda$, and $\beta$.

Proof. We have

$$
\operatorname{div}\left(A \nabla v_{\varepsilon}\right)+\varepsilon^{2} k^{2} v_{\varepsilon}=f+\varepsilon^{2} k^{2} v_{\varepsilon}-\varepsilon^{2} k^{2} \Sigma_{\varepsilon} v_{\varepsilon} .
$$

From Lemma 4, we deduce that

$$
\left\|v_{\varepsilon}\right\|_{L^{2}\left(B_{5}\right)} \leq C|\ln (k \varepsilon)|^{2}\left(\|f\|_{L^{2}}+\varepsilon^{2} k^{2}\left\|v_{\varepsilon}\right\|_{L^{2}\left(B_{5}\right)}+\frac{k \varepsilon}{\lambda}\left\|v_{\varepsilon}\right\|_{L^{2}\left(B_{5}\right)}+k^{2}\left\|v_{\varepsilon}\right\|_{L^{2}\left(B_{5}\right)}\right) .
$$

By selecting $c$ sufficiently small and using the facts that $\varepsilon^{2} k^{2}|\ln (\varepsilon k)|^{2} \leq c, \varepsilon k|\ln (\varepsilon k)|^{2}$ $\leq c \lambda$, and $k^{2}|\ln (\varepsilon k)|^{2} \leq c$, the last three terms on the right-hand side may be absorbed by (half) the left-hand side, and we arrive at the first estimate (2.38).

The second estimate (2.39) follows from a combination of (2.38) and Proposition 2 .

3. Proof of the main results. Let $u_{\varepsilon}$ be the unique solution of the wave equation

$$
\begin{cases}\Sigma_{1, \varepsilon} \partial_{t t}^{2} u_{\varepsilon}-\operatorname{div}\left(A_{\varepsilon} \nabla u_{\varepsilon}\right)+\Sigma_{2, \varepsilon} \partial_{t} u_{\varepsilon}=f & \text { in } \mathbb{R}_{+} \times \mathbb{R}^{d}, \\ u_{\varepsilon}(t=0)=u_{0} & \text { in } \mathbb{R}^{d}, \\ \partial_{t} u_{\varepsilon}(t=0)=u_{1} & \text { in } \mathbb{R}^{d},\end{cases}
$$

where $A_{\varepsilon}, \Sigma_{1, \varepsilon}, \Sigma_{2, \varepsilon}, 0<\varepsilon<1$ are time independent and defined as

$$
A_{\varepsilon}, \Sigma_{1, \varepsilon}, \Sigma_{2, \varepsilon}= \begin{cases}I, 1,0 & \text { in } \mathbb{R}^{3} \backslash B_{\varepsilon}, \\ I, 1,1 / \varepsilon^{2+\gamma} & \text { in } B_{\varepsilon} \backslash B_{\varepsilon / 2}, \\ F_{\varepsilon}^{-1}{ }_{*} a, F_{\varepsilon}^{-1}{ }_{*} \sigma, 0 & \text { in } B_{\varepsilon / 2}\end{cases}
$$

with $a, \sigma$ satisfying (1.5). By direct computation, $A_{c}, \Sigma_{1, c}, \Sigma_{2, c}=F_{\varepsilon_{*}} A_{\varepsilon}, F_{\varepsilon_{*}} \Sigma_{1, \varepsilon}, F_{\varepsilon_{*}}$ $\Sigma_{2, \varepsilon}$. Hence, using the invariance of the wave equation under change of variables and the fact that $u_{0}, u_{1}$, and $f$ vanish for $|x|<2$, we have

$$
u_{c}(t, x)=u_{\varepsilon}\left(t, F_{\varepsilon}^{-1}(x)\right) \text {, }
$$

and so

$$
u_{c}(t, x)=u_{\varepsilon}(t, x) \quad \text { for } x \in \mathbb{R}^{d} \backslash B_{2} .
$$

The main theorems, Theorems 1 and 2, are now consequences of the following results.

Copyright $@$ by SIAM. Unauthorized reproduction of this article is prohibited. 
TheOrem 3. Let $d=3$ and $\gamma>0$. Given $R>2$, there exists a positive constant $C$ depending only on $\Lambda, \gamma$, and $R$ such that

$$
\sup _{t>0}\left\|u_{\varepsilon}(t, \cdot)-u(t, \cdot)\right\|_{L^{2}\left(B_{R} \backslash B_{2}\right)} \leq C \varepsilon\left(\|f\|+\left\|u_{0}\right\|+\left\|u_{1}\right\|\right),
$$

where $u$ is the solution to (1.3).

TheOREM 4. Let $d=2$ and $\gamma>0$. Given $R>2$, there exists a positive constant $C$ depending only on $\Lambda, \gamma$, and $R$ such that

$$
\sup _{t>0}\left\|u_{\varepsilon}(t, \cdot)-u(t, \cdot)\right\|_{L^{2}\left(B_{R} \backslash B_{2}\right)} \leq \frac{C}{|\ln \varepsilon|}\left(\|f\|+\left\|u_{0}\right\|+\left\|u_{1}\right\|\right),
$$

where $u$ is the solution to (1.3).

3.1. Proof of Theorem 3. We split $u_{\varepsilon}-u$ into two parts. To this end, consider $\tilde{u}_{\varepsilon} \in H_{\text {loc }}^{1}\left(\mathbb{R}_{+} ; H^{1}\left(\mathbb{R}^{3}\right)\right)$, uniquely determined by

$$
\begin{cases}\partial_{t t}^{2} \tilde{u}_{\varepsilon}-\Delta \tilde{u}_{\varepsilon}=f & \text { in } \mathbb{R}_{+} \times\left(\mathbb{R}^{3} \backslash \bar{B}_{\varepsilon}\right), \\ \tilde{u}_{\varepsilon}=0 & \text { in } \mathbb{R}_{+} \times B_{\varepsilon} \\ \tilde{u}_{\varepsilon}(t=0)=u_{0} & \text { in } \mathbb{R}^{3} \\ \partial_{t} \tilde{u}_{\varepsilon}(t=0)=u_{1} & \text { in } \mathbb{R}^{3}\end{cases}
$$

and set

$$
\left\{\begin{array}{l}
v_{\varepsilon}=\tilde{u}_{\varepsilon}-u \\
w_{\varepsilon}=u_{\varepsilon}-\tilde{u}_{\varepsilon}
\end{array}\right.
$$

We proceed to show that

$$
\sup _{t>0}\left\|v_{\varepsilon}(t, \cdot)\right\|_{L^{2}\left(B_{R} \backslash B_{2}\right)} \leq C \varepsilon \text { Data }
$$

and

$$
\sup _{t>0}\left\|w_{\varepsilon}(t, \cdot)\right\|_{L^{2}\left(B_{R} \backslash B_{2}\right)} \leq C \varepsilon \text { Data }
$$

where

$$
\text { Data }=\|f\|+\left\|u_{0}\right\|+\left\|u_{1}\right\| .
$$

Here and in the following, $C$ denotes a positive constant depending only on $\Lambda, \gamma$, and $R$. Since $u_{\varepsilon}-u=v_{\varepsilon}+w_{\varepsilon}$, the inequalities (3.3) and (3.4) are sufficient to obtain the estimate of Theorem 3.

Step 1: Proof of (3.3). From the definition of $v_{\varepsilon}$ in (3.2), we have

$$
\begin{cases}\partial_{t t}^{2} v_{\varepsilon}-\Delta v_{\varepsilon}=0 & \text { in } \mathbb{R}_{+} \times\left(\mathbb{R}^{3} \backslash \bar{B}_{\varepsilon}\right), \\ v_{\varepsilon}=-u & \text { on } \mathbb{R}_{+} \times \partial B_{\varepsilon}, \\ v_{\varepsilon}(t=0)=\partial_{t} v_{\varepsilon}(t=0)=0 . & \end{cases}
$$

Let $\hat{v}_{\varepsilon}(k, x)$ denote the Fourier transform (in time) of $v_{\varepsilon}(t, x)$. In this paper, by the Fourier transform of a function defined on $[0, \infty)$, we mean the Fourier transform of

Copyright (c) by SIAM. Unauthorized reproduction of this article is prohibited. 
the extension by 0 for negative time. We claim that $\hat{v}_{\varepsilon}(k, \cdot)$ satisfies the outgoing radiation condition. To see this, let $\phi \in C^{\infty}\left(\mathbb{R}^{3}\right)$ be such that $\phi(x)=0$ on $B_{1}$ and $\phi(x)=1$ for $|x|>2$. Set $\xi=\tilde{u}_{\varepsilon}-u \phi$. We have

$$
\begin{cases}\partial_{t t}^{2} \xi-\Delta \xi=u \Delta \phi+2 \nabla u \nabla \phi & \text { in } \mathbb{R}_{+} \times\left(\mathbb{R}^{3} \backslash \bar{B}_{\varepsilon}\right), \\ \xi=0 & \text { on } \mathbb{R}_{+} \times \partial B_{\varepsilon}, \\ \xi(t=0)=\partial_{t} \xi(t=0)=0 & \end{cases}
$$

for $\varepsilon<1$. Applying Huyghen's principle and Theorem A2, we conclude that $\hat{\xi}(k, \cdot)$ satisfies the outgoing radiation condition. Since $\hat{v}_{\varepsilon}(k, x)=\hat{\xi}(k, x)$ for $|x|>2, \hat{v}_{\varepsilon}(k, \cdot)$ also satisfies the outgoing radiation condition. Thus $\hat{v}_{\varepsilon}(k, \cdot) \in H_{l o c}^{1}\left(\mathbb{R}^{3} \backslash B_{\varepsilon}\right)$ is the unique outgoing solution to

$$
\begin{cases}\Delta \hat{v}_{\varepsilon}(k, \cdot)+k^{2} \hat{v}_{\varepsilon}(k, \cdot)=0 & \text { in } \mathbb{R}^{3} \backslash \bar{B}_{\varepsilon}, \\ \hat{v}_{\varepsilon}(k, \cdot)=-\hat{u}(k, \cdot) & \text { on } \partial B_{\varepsilon}\end{cases}
$$

for almost all $k>0$. Set

$$
\hat{V}_{\varepsilon}(k, x)=\hat{v}_{\varepsilon}(k, \varepsilon x) .
$$

It follows that

$$
\begin{cases}\Delta \hat{V}_{\varepsilon}(k, \cdot)+k^{2} \varepsilon^{2} \hat{V}_{\varepsilon}(k, \cdot)=0 & \text { in } \mathbb{R}^{3} \backslash \bar{B}_{1}, \\ \hat{V}_{\varepsilon}(k, \cdot)=-\hat{u}(k, \varepsilon \cdot) & \text { on } \partial B_{1}\end{cases}
$$

for almost all $k>0$. Applying propositions 1 and 2, we have

$$
\varepsilon \int_{B_{R / \varepsilon} \backslash B_{2 / \varepsilon}}\left|\hat{V}_{\varepsilon}(k, \cdot)\right|^{2} \leq C\|\hat{u}(k, \varepsilon \cdot)\|_{H^{1}\left(\partial B_{1}\right)}^{2} \quad \text { for } k>0
$$

and

$$
\int_{B_{R} \backslash B_{1}}\left(\left|\nabla \hat{V}_{\varepsilon}(k, x)\right|^{2}+\left|\hat{V}_{\varepsilon}(k, x)\right|^{2}\right) d x \leq C\|\hat{u}(k, \varepsilon \cdot)\|_{H^{1}\left(\partial B_{1}\right)}^{2} \quad \text { for } k>0 .
$$

Let $\hat{u}(k, \cdot)$ be the Fourier transform (in time) of $u$. Since $u(\cdot, x) \in L^{1}\left(\mathbb{R}_{+}\right)$(due to Huyghen's principle), we have

$$
\hat{u}(k, x)=\frac{1}{\sqrt{2 \pi}} \int_{0}^{\infty} e^{i t k} u(t, x) d t .
$$

As a consequence of (3.7) and the fact that $u(t=0, x)=\partial_{t} u(t=0, x)=0$ for $x \in B_{1}$,

$$
\begin{aligned}
& \left(1+k^{2}\right)(|\hat{u}(k, x)|+|\nabla \hat{u}(k, x)|) \\
& \leq \int_{0}^{\infty}\left(|u(t, x)|+\left|\partial_{t t} u(t, x)\right|+|\nabla u(t, x)|+\left|\partial_{t t} \nabla u(t, x)\right|\right) d t \quad \text { for } x \in B_{1} .
\end{aligned}
$$

This implies

$$
\left(1+k^{2}\right)(|\hat{u}(k, x)|+|\nabla \hat{u}(k, x)|) \leq C D a t a \quad \text { for } x \in B_{1} .
$$

Copyright $@$ by SIAM. Unauthorized reproduction of this article is prohibited. 
It follows from (3.5), (3.6), and (3.8) that

$$
\varepsilon \int_{B_{R / \varepsilon} \backslash B_{2 / \varepsilon}}\left|\hat{V}_{\varepsilon}(k, \cdot)\right|^{2} \leq \frac{C}{1+k^{4}} \text { Data }^{2} \quad \text { for } k>0
$$

and

$$
\int_{B_{R} \backslash B_{1}}\left(\left|\nabla \hat{V}_{\varepsilon}(k, x)\right|^{2}+\left|\hat{V}_{\varepsilon}(k, x)\right|^{2}\right) d x \leq \frac{C}{1+k^{4}} \text { Data }^{2} .
$$

This implies

$$
\sqrt{\varepsilon} \int_{0}^{\infty}\left\|\hat{V}_{\varepsilon}(k, \cdot)\right\|_{L^{2}\left(B_{R / \varepsilon} \backslash B_{2 / \varepsilon}\right)} d k \leq C D a t a,
$$

and by a change of variable,

$$
\int_{0}^{\infty}\left\|\hat{v}_{\varepsilon}(k, \cdot)\right\|_{L^{1}\left(B_{R} \backslash B_{2}\right)} d k \leq C \int_{0}^{\infty}\left\|\hat{v}_{\varepsilon}(k, \cdot)\right\|_{L^{2}\left(B_{R} \backslash B_{2}\right)} d k \leq C \varepsilon \text { Data. }
$$

Hence $\hat{v}(\cdot, x) \in L^{1}\left(\mathbb{R}_{+}\right)$for almost all $x \in \mathbb{R}^{3}$, and by the inversion formula

$$
v_{\varepsilon}(t, x)=2 \Re\left\{\frac{1}{\sqrt{2 \pi}} \int_{0}^{\infty} \hat{v}_{\varepsilon}(k, x) e^{-i k t} d k\right\},
$$

we obtain ${ }^{4}$

$$
\sup _{t>0}\left\|v_{\varepsilon}(t, \cdot)\right\|_{L^{2}\left(B_{R} \backslash B_{2}\right)} \leq C \varepsilon \text { Data. }
$$

Step 2: Proof of (3.4). From the definition of $w_{\varepsilon}$ in (3.2), we have

$$
\begin{cases}\Sigma_{1, \varepsilon} \partial_{t t}^{2} w_{\varepsilon}-\operatorname{div}\left(A_{\varepsilon} \nabla w_{\varepsilon}\right)+\Sigma_{2, \varepsilon} \partial_{t} w_{\varepsilon}=0 & \text { in } \mathbb{R}_{+} \times\left(\mathbb{R}^{3} \backslash \partial B_{\varepsilon}\right), \\ {\left[w_{\varepsilon}\right]=0 \text { and }\left[\partial_{r} w_{\varepsilon}\right]=-\left.\partial_{r} \tilde{u}_{\varepsilon}\right|_{\text {ext }}=-\left.\partial_{r}\left(v_{\varepsilon}+u\right)\right|_{\text {ext }}} & \text { on } \mathbb{R}_{+} \times \partial B_{\varepsilon}, \\ w_{\varepsilon}(t=0)=\partial_{t} w_{\varepsilon}(t=0)=0 . & \end{cases}
$$

Let $\hat{w}_{\varepsilon}(\cdot, x)$ denote the Fourier transform of $w_{\varepsilon}(\cdot, x)$. We have $\hat{w}_{\varepsilon}=\left(\hat{u}_{\varepsilon}-\hat{u}\right)-\hat{v}_{\varepsilon}$. By Theorem A2, $\hat{v}_{\varepsilon}(k, \cdot)$ satisfies the outgoing radiation condition. We claim that $\left(\hat{u}_{\varepsilon}-\hat{u}\right)(k, \cdot)$ also satisfies the outgoing radiation condition. To see this, let $\phi \in$ $C^{\infty}\left(\mathbb{R}^{3}\right)$ be such that $\phi(x)=0$ on $B_{1}$ and $\phi(x)=1$ for $|x|>2$. Set $\xi=u_{\varepsilon}-u \phi$. We have

$$
\begin{cases}\Sigma_{1, \varepsilon} \partial_{t t}^{2} \xi-\operatorname{div}\left(A_{\varepsilon} \nabla \xi\right)+\Sigma_{2, \varepsilon} \partial_{t} \xi=u \Delta \phi+2 \nabla u \nabla \phi & \text { in } \mathbb{R}_{+} \times \mathbb{R}^{3}, \\ \xi(t=0)=\partial_{t} \xi(t=0)=0 & \text { in } \mathbb{R}^{3}\end{cases}
$$

for $\varepsilon<1$. Using Huyghen's principle and Theorem A1, we obtain that $\hat{\xi}(k, \cdot)$ satisfies the outgoing radiation condition. Since $\hat{\xi}(k, x)=\hat{u}_{\varepsilon}(k, x)-\hat{u}(k, x)$ for $|x|>2$, the claim is proved. As a consequence $\hat{w}_{\varepsilon}(k, \cdot)$ satisfies the outgoing radiation condition. It follows that $\hat{w}_{\varepsilon}(k, \cdot) \in H_{\text {loc }}^{1}\left(\mathbb{R}^{3}\right)$ is the unique outgoing solution to

$$
\begin{cases}\operatorname{div}\left(A_{\varepsilon} \nabla \hat{w}_{\varepsilon}(k, \cdot)\right)+\Sigma_{\varepsilon} k^{2} \hat{w}_{\varepsilon}(k, \cdot)=0 & \text { in } \mathbb{R}^{3} \backslash \partial B_{\varepsilon}, \\ {\left[\partial_{r} \hat{w}_{\varepsilon}(k, \cdot)\right]=-\left.\partial_{r}\left(\hat{v}_{\varepsilon}+\hat{u}\right)(k, \cdot)\right|_{\text {ext }}} & \text { on } \partial B_{\varepsilon}\end{cases}
$$

\footnotetext{
${ }^{4}$ Using the fact that $\left\|\int_{0}^{\infty} \phi(k) d k\right\|_{L^{2}} \leq \int_{0}^{\infty}\|\phi(k)\|_{L^{2}} d k$.
}

Copyright $@$ by SIAM. Unauthorized reproduction of this article is prohibited. 
for almost all $k>0$. Here

$$
\Sigma_{\varepsilon}=\Sigma_{1, \varepsilon}+\frac{i}{k} \Sigma_{2, \varepsilon}
$$

Define $\hat{W}_{\varepsilon}(k, x)=\hat{w}_{\varepsilon}(k, \varepsilon x)$ and $\hat{U}_{\varepsilon}(k, x)=\hat{u}(k, \varepsilon x)$. It follows that $\hat{W}_{\varepsilon}(k, \cdot) \in$ $H_{\text {loc }}^{1}\left(\mathbb{R}^{3}\right)$ is the unique outgoing solution of

$$
\begin{cases}\operatorname{div}\left(A_{\varepsilon}(\varepsilon x) \nabla \hat{W}_{\varepsilon}(k, \cdot)\right)+\varepsilon^{2} k^{2} \Sigma_{\varepsilon}(\varepsilon x) \hat{W}_{\varepsilon}(k, \cdot)=0 & \text { in } \mathbb{R}^{3} \backslash \partial B_{1}, \\ {\left[\partial_{r} \hat{W}_{\varepsilon}(k, \cdot)\right]=-\partial_{r} \hat{V}_{\varepsilon}(k, \cdot)-\left.\partial_{r} \hat{U}_{\varepsilon}(k, \cdot)\right|_{\text {ext }}} & \text { on } \partial B_{1}\end{cases}
$$

for almost all $k>0$. From (3.8), we have

$$
\int_{B_{4}}\left(\left|\nabla \hat{U}_{\varepsilon}(k, x)\right|^{2}+\left|\hat{U}_{\varepsilon}(k, x)\right|^{2}\right) d x \leq \frac{C}{1+k^{4}} D a t a^{2}
$$

for $\varepsilon<1 / 4$. Using (3.10) and (3.13) in combination with Proposition 3, we obtain

$$
\varepsilon \int_{B_{R / \varepsilon} \backslash B_{2 / \varepsilon}}\left|\hat{W}_{\varepsilon}(k, \cdot)\right|^{2} \leq \frac{C}{1+k^{4}} \text { Data }^{2} \quad \text { for } k \geq \varepsilon^{\gamma}
$$

and

$$
\varepsilon \int_{B_{R / \varepsilon} \backslash B_{2 / \varepsilon}}\left|\hat{W}_{\varepsilon}(k, \cdot)\right|^{2} \leq \frac{C \varepsilon^{2 \gamma}}{k^{2}} \operatorname{Data}^{2} \quad \text { for } c \min \left\{\varepsilon^{1 / 2}, \varepsilon^{\gamma}\right\} \leq k \leq \varepsilon^{\gamma} .
$$

Using (3.10) and (3.13) in combination with Proposition 5, we obtain

$$
\varepsilon \int_{B_{R / \varepsilon} \backslash B_{2 / \varepsilon}}\left|\hat{W}_{\varepsilon}(k, \cdot)\right|^{2} \leq C \text { Data }^{2} \quad \text { for } 0<k \leq c \min \left\{\varepsilon^{1 / 2}, \varepsilon^{\gamma}\right\} .
$$

We therefore have

$$
\begin{aligned}
\sqrt{\varepsilon} \int_{0}^{\infty}\left\|\hat{W}_{\varepsilon}(k, \cdot)\right\|_{L^{2}\left(B_{R / \varepsilon} \backslash B_{2 / \varepsilon}\right)} d k \\
\quad=\left(\int_{\varepsilon^{\gamma}}^{\infty}+\int_{c \min \left\{\varepsilon^{1 / 2}, \varepsilon^{\gamma}\right\}}^{\varepsilon^{\gamma}}+\int_{0}^{c \min \left\{\varepsilon^{1 / 2}, \varepsilon^{\gamma}\right\}}\right) \sqrt{\varepsilon}\left\|\hat{W}_{\varepsilon}(k, \cdot)\right\|_{L^{2}\left(B_{R / \varepsilon} \backslash B_{2 / \varepsilon}\right)} d k \\
\leq C\left(\text { Data }+ \text { Data } \varepsilon^{\gamma} \int_{c \min \left\{\varepsilon^{1 / 2}, \varepsilon^{\gamma}\right\}}^{\varepsilon^{\gamma}} \frac{1}{k}+\text { Data } \min \left\{\varepsilon^{1 / 2}, \varepsilon^{\gamma}\right\}\right) .
\end{aligned}
$$

Since $\gamma>0$, this implies

$$
\sqrt{\varepsilon} \int_{0}^{\infty}\left\|\hat{W}_{\varepsilon}(k, \cdot)\right\|_{L^{2}\left(B_{R / \varepsilon} \backslash B_{2 / \varepsilon}\right)} d k \leq \text { Data },
$$

and by a change of variables,

$$
\int_{0}^{\infty}\left\|\hat{w}_{\varepsilon}(k, \cdot)\right\|_{L^{2}\left(B_{R} \backslash B_{2}\right)} d k \leq C \varepsilon \text { Data. }
$$

Hence $\hat{w}_{\varepsilon}(\cdot, x) \in L^{1}\left(\mathbb{R}_{+}\right)$for almost all $x$, and due to the inversion formula

$$
w_{\varepsilon}(t, x)=2 \Re\left\{\frac{1}{\sqrt{2 \pi}} \int_{0}^{\infty} \hat{w}_{\varepsilon}(k, x) e^{-i k t} d k\right\}
$$

we conclude that

$$
\sup _{t>0}\left\|w_{\varepsilon}(t, \cdot)\right\|_{L^{2}\left(B_{R} \backslash B_{2}\right)} \leq C \varepsilon \text { Data. }
$$

Copyright $@$ ( ) by SIAM. Unauthorized reproduction of this article is prohibited. 
3.2. Proof of Theorem 4. We follow the strategy used in the proof of Theorem 3. We split $u_{\varepsilon}-u$ into two parts. To this end, consider $\tilde{u}_{\varepsilon} \in H_{l o c}^{1}\left(\mathbb{R}_{+}, H^{1}\left(\mathbb{R}^{2}\right)\right)$ uniquely determined by

$$
\begin{cases}\partial_{t t}^{2} \tilde{u}_{\varepsilon}-\Delta \tilde{u}_{\varepsilon}=f & \text { in } \mathbb{R}_{+} \times\left(\mathbb{R}^{2} \backslash \bar{B}_{\varepsilon}\right), \\ \tilde{u}_{\varepsilon}=0 & \text { in } \mathbb{R}_{+} \times B_{\varepsilon} \\ \tilde{u}_{\varepsilon}(t=0)=u_{0} & \text { in } \mathbb{R}^{2} \\ \partial_{t} \tilde{u}_{\varepsilon}(t=0)=u_{1} & \text { in } \mathbb{R}^{2}\end{cases}
$$

and set

$$
\left\{\begin{array}{l}
v_{\varepsilon}=\tilde{u}_{\varepsilon}-u \\
w_{\varepsilon}=u_{\varepsilon}-\tilde{u}_{\varepsilon}
\end{array}\right.
$$

We proceed to show that

$$
\sup _{t>0}\left\|v_{\varepsilon}(t, \cdot)\right\|_{L^{2}\left(B_{R} \backslash B_{2}\right)} \leq \frac{C}{|\ln \varepsilon|} \text { Data }
$$

and

$$
\sup _{t>0}\left\|w_{\varepsilon}(t, \cdot)\right\|_{L^{2}\left(B_{R} \backslash B_{2}\right)} \leq \frac{C}{|\ln \varepsilon|} \text { Data }
$$

where

$$
\text { Data }=\|f\|+\left\|u_{0}\right\|+\left\|u_{1}\right\| .
$$

Here and in the following, $C$ denotes a positive constant depending only on $\Lambda$, $\gamma$, and $R$. Since $u_{\varepsilon}-u=v_{\varepsilon}+w_{\varepsilon}$, the inequalities (3.15) and (3.16) are sufficient to obtain the estimate of Theorem 4.

Step 1: Proof of (3.15). From the definition of $v_{\varepsilon}$ in (3.14), we have

$$
\begin{cases}\partial_{t t}^{2} v_{\varepsilon}-\Delta v_{\varepsilon}=0 & \text { in } \mathbb{R}_{+} \times\left(\mathbb{R}^{2} \backslash \bar{B}_{\varepsilon}\right), \\ v_{\varepsilon}=-u & \text { on } \mathbb{R}_{+} \times \partial B_{\varepsilon}, \\ v_{\varepsilon}(t=0)=\partial_{t} v_{\varepsilon}(t=0)=0 . & \end{cases}
$$

Let $\hat{v}_{\varepsilon}(k, x)$ be the Fourier transform of $v_{\varepsilon}(\cdot, x)$ with respect to time. We claim that $\hat{v}_{\varepsilon}(k, \cdot)$ satisfies the outgoing radiation condition. To see this, let $\phi \in C^{\infty}\left(\mathbb{R}^{2}\right)$ be such that $\phi(x)=0$ on $B_{1}$ and $\phi(x)=1$ for $|x|>2$. Set $\xi=\tilde{u}_{\varepsilon}-u \phi$. We have

$$
\begin{cases}\partial_{t t}^{2} \xi-\Delta \xi=u \Delta \phi+2 \nabla u \nabla \phi & \text { in } \mathbb{R}_{+} \times\left(\mathbb{R}^{2} \backslash \bar{B}_{\varepsilon}\right), \\ \xi=0 & \text { on } \mathbb{R}_{+} \times \partial B_{\varepsilon} \\ \xi(t=0)=\partial_{t} \xi(t=0)=0 & \end{cases}
$$

for $\varepsilon<1$. Applying theorems A2 and B1, we conclude that $\hat{\xi}(k, \cdot)$ satisfies the outgoing radiation condition. Since $\hat{v}_{\varepsilon}(k, x)=\hat{\xi}(k, x)$ for $|x|>2, \hat{v}_{\varepsilon}(k, \cdot)$ also satisfies the outgoing radiation condition. Thus $\hat{v}_{\varepsilon}(k, \cdot) \in H_{\text {loc }}^{1}\left(\mathbb{R}^{2} \backslash B_{\varepsilon}\right)$ is the unique outgoing solution to

$$
\begin{cases}\Delta \hat{v}_{\varepsilon}(k, \cdot)+k^{2} \hat{v}_{\varepsilon}(k, \cdot)=0 & \text { in } \mathbb{R}^{2} \backslash \bar{B}_{\varepsilon} \\ \hat{v}_{\varepsilon}(k, \cdot)=-\hat{u}(k, \cdot) & \text { on } \partial B_{\varepsilon}\end{cases}
$$

Copyright $@$ ㅇ by SIAM. Unauthorized reproduction of this article is prohibited. 
for almost all $k>0$. Here $\hat{u}$ is the Fourier transform of $u$ with respect to time. Set

$$
\hat{V}_{\varepsilon}(k, x)=\hat{v}_{\varepsilon}(k, \varepsilon x) .
$$

It follows that $\hat{V}_{\varepsilon}(k, \cdot) \in H_{l o c}^{1}\left(\mathbb{R}^{2} \backslash B_{1}\right)$ is the unique outgoing solution to

$$
\begin{cases}\Delta \hat{V}_{\varepsilon}(k, \cdot)+\varepsilon^{2} k^{2} \hat{V}_{\varepsilon}(k, \cdot)=0 & \text { in } \mathbb{R}^{2} \backslash \bar{B}_{1} \\ \hat{V}_{\varepsilon}(k, \cdot)=-\hat{u}(k, \varepsilon \cdot) & \text { on } \partial B_{1}\end{cases}
$$

for almost all $k>0$. Applying Propositions 1 and 2, we have

$$
\varepsilon^{2} \int_{B_{R / \varepsilon} \backslash B_{2 / \varepsilon}}\left|\hat{V}_{\varepsilon}(k, \cdot)\right|^{2} \leq C \varepsilon\|\hat{u}(k, \varepsilon \cdot)\|_{H^{1}\left(\partial B_{1}\right)}^{2} \quad \text { for } k \geq 1 /(2 \varepsilon),
$$

$$
\varepsilon^{2} \int_{B_{R / \varepsilon} \backslash B_{2 / \varepsilon}}\left|\hat{V}_{\varepsilon}(k, \cdot)\right|^{2} \leq \frac{C\left|H_{1}^{(0)}(k)\right|^{2}}{|\ln (\varepsilon k)|^{2}}\|\hat{u}(k, \varepsilon \cdot)\|_{H^{1 / 2}\left(\partial B_{1}\right)}^{2} \quad \text { for } 0<k \leq 1 /(2 \varepsilon),
$$

and

$$
\int_{B_{R} \backslash B_{1}}\left(\left|\nabla \hat{V}_{\varepsilon}(k, x)\right|^{2}+\left|\hat{V}_{\varepsilon}(k, x)\right|^{2}\right) d x \leq C\|\hat{u}(k, \varepsilon \cdot)\|_{H^{1}\left(\partial B_{1}\right)}^{2}
$$

for $k>0$. By Placherel's theorem, we have

$$
\int_{0}^{\infty}\left(1+k^{2}\right)\|\hat{u}(k, \cdot)\|_{H^{3}\left(B_{1}\right)}^{2} d k \leq \int_{0}^{\infty}\left(\|u(t, \cdot)\|_{H^{3}\left(B_{1}\right)}^{2}+\left\|\partial_{t} u(t, \cdot)\right\|_{H^{3}\left(B_{1}\right)}^{2}\right) d t,
$$

since $u(t=0, x)=0$ for $x \in B_{1}$. Applying Theorem B1 and Remark 6 (in Appendix B), we obtain

$$
\int_{0}^{\infty}\left(1+k^{2}\right)\|\hat{u}(k, \cdot)\|_{W^{1, \infty}\left(B_{1}\right)}^{2} \leq C \text { Data }^{2} .
$$

A combination of (3.17), (3.18), and (3.20) yields

$$
\varepsilon \int_{0}^{\infty}\left\|\hat{V}_{\varepsilon}(k, \cdot)\right\|_{L^{2}\left(B_{R / \varepsilon} \backslash B_{2 / \varepsilon}\right)} d k \leq \frac{C D a t a}{|\ln \varepsilon|} .
$$

Here we use the fact that

$$
\frac{\left|H_{0}^{(1)}(k)\right|}{|\ln (\varepsilon k)|} \leq \frac{C}{|\ln \varepsilon|}(|\ln k|+1), \quad 0<k<1 /(2 \varepsilon),
$$

which follows from

$$
\frac{\left|H_{0}^{(1)}(k)\right|}{|\ln (\varepsilon k)|} \leq C\left\{\begin{array}{cc}
\frac{|\ln k|+1}{|\ln \varepsilon|} & \text { if } 0<k<\frac{1}{2 \sqrt{\varepsilon}} \\
\varepsilon^{1 / 4} & \text { if } \frac{1}{2 \sqrt{\varepsilon}}<k<\frac{1}{2 \varepsilon} .
\end{array}\right.
$$

After a change of variables, (3.21) yields

$$
\int_{0}^{\infty}\left\|\hat{v}_{\varepsilon}(k, \cdot)\right\|_{L^{2}\left(B_{R} \backslash B_{2}\right)} d k \leq \frac{C \text { Data }}{|\ln \varepsilon|} .
$$

Copyright $@$ by SIAM. Unauthorized reproduction of this article is prohibited. 
Hence $\hat{v}_{\varepsilon}(\cdot, x) \in L^{1}\left(\mathbb{R}_{+}\right)$for almost all $x \in \mathbb{R}^{2}$, and by the inversion formula

$$
v_{\varepsilon}(t, x)=2 \Re\left\{\frac{1}{\sqrt{2 \pi}} \int_{0}^{\infty} \hat{v}_{\varepsilon}(k, x) e^{-i k t} d k\right\},
$$

we obtain (3.15).

Step 2: Proof of (3.16). From the definition of $w_{\varepsilon}$ in (3.14), we have

$$
\begin{cases}\Sigma_{1, \varepsilon} \partial_{t t}^{2} w_{\varepsilon}-\operatorname{div}\left(A_{\varepsilon} \nabla w_{\varepsilon}\right)+\Sigma_{2, \varepsilon} \partial_{t} w_{\varepsilon}=0 & \text { in } \mathbb{R}_{+} \times\left(\mathbb{R}^{2} \backslash \partial B_{\varepsilon}\right), \\ {\left[w_{\varepsilon}\right]=0 \text { and }\left[\partial_{r} w_{\varepsilon}\right]=-\left.\partial_{r} \tilde{u}_{\varepsilon}\right|_{\text {ext }}=-\left.\partial_{r}\left(v_{\varepsilon}+u\right)\right|_{\text {ext }}} & \text { on } \mathbb{R}_{+} \times \partial B_{\varepsilon}, \\ w_{\varepsilon}(t=0)=\partial_{t} w_{\varepsilon}(t=0)=0 . & \end{cases}
$$

Let $\hat{w}_{\varepsilon}(\cdot, x)$ denote the Fourier transform of $w_{\varepsilon}(\cdot, x)$. We have $\hat{w}_{\varepsilon}=\left(\hat{u}_{\varepsilon}-\hat{u}\right)-\hat{v}_{\varepsilon}$. By Theorem A2, $\hat{v}_{\varepsilon}$ satisfies the outgoing radiation condition. We claim that $\hat{u}_{\varepsilon}-\hat{u}$ also satisfies the outgoing radiation condition. To see this, let $\phi \in C^{\infty}\left(\mathbb{R}^{2}\right)$ be such that $\phi(x)=0$ on $B_{1}$ and $\phi(x)=1$ for $|x|>2$. Set $\xi=u_{\varepsilon}-u \phi$. We have

$$
\begin{cases}\Sigma_{1, \varepsilon} \partial_{t t}^{2} \xi-\operatorname{div}\left(A_{\varepsilon} \nabla \xi\right)+\Sigma_{2, \varepsilon} \partial_{t} \xi=u \Delta \phi+2 \nabla u \nabla \phi & \text { in } \mathbb{R}_{+} \times \mathbb{R}^{2} \\ \xi(t=0)=\partial_{t} \xi(t=0)=0 & \text { in } \mathbb{R}^{2}\end{cases}
$$

for $\varepsilon<1$. Applying Theorems A1 and B1, we conclude that $\hat{\xi}(k, \cdot)$ satisfies the outgoing radiation condition. Since $\hat{u}_{\varepsilon}-\hat{u}=\hat{\xi}$ for $|x|>2, \hat{u}_{\varepsilon}-\hat{u}$ satisfies the outgoing radiation condition. As a consequence $\hat{w}_{\varepsilon}(k, \cdot)$ satisfies the outgoing radiation condition. It follows that $\hat{w}_{\varepsilon}(k, \cdot) \in H_{l o c}^{1}\left(\mathbb{R}^{2}\right)$ is the unique outgoing solution to

$$
\begin{cases}\operatorname{div}\left(A_{\varepsilon} \nabla \hat{w}_{\varepsilon}(k, \cdot)\right)+k^{2} \Sigma_{\varepsilon} \hat{w}_{\varepsilon}(k, \cdot)=0 & \text { in } \mathbb{R}^{2} \backslash \partial B_{\varepsilon}, \\ {\left[\partial_{r} \hat{w}_{\varepsilon}(k, \cdot)\right]=-\left.\partial_{r}\left(\hat{v}_{\varepsilon}+\hat{u}\right)(k, \cdot)\right|_{\text {ext }}} & \text { on } \partial B_{\varepsilon}\end{cases}
$$

for almost all $k>0$. Here

$$
\Sigma_{\varepsilon}=\Sigma_{1, \varepsilon}+\frac{i}{k} \Sigma_{2, \varepsilon}
$$

Define $\hat{W}_{\varepsilon}(k, x)=\hat{w}_{\varepsilon}(k, \varepsilon x)$ and $\hat{U}_{\varepsilon}(k, x)=\hat{u}(k, \varepsilon x)$. It follows that $\hat{W}_{\varepsilon}(k, \cdot) \in$ $H_{\text {loc }}^{1}\left(\mathbb{R}^{2}\right)$ is the unique outgoing solution to

$$
\begin{cases}\operatorname{div}\left(A_{\varepsilon}(\varepsilon x) \nabla \hat{W}_{\varepsilon}(k, \cdot)\right)+\varepsilon^{2} k^{2} \Sigma_{\varepsilon}(\varepsilon x) \hat{W}_{\varepsilon}(k, \cdot)=0 & \text { in } \mathbb{R}^{2} \backslash \partial B_{1}, \\ {\left[\partial_{r} \hat{W}_{\varepsilon}(k, \cdot)\right]=-\partial_{r} \hat{V}_{\varepsilon}(k, \cdot)-\left.\partial_{r} \hat{U}_{\varepsilon}(k, \cdot)\right|_{\text {ext }}} & \text { on } \partial B_{1} .\end{cases}
$$

We have (for $\varepsilon<1 / 4$ )

$$
\int_{B_{4}}\left(\left|\nabla \hat{U}_{\varepsilon}(k, x)\right|^{2}+\left|\hat{U}_{\varepsilon}(k, x)\right|^{2}\right) d x \leq C\|\hat{u}(k, \cdot)\|_{W^{1, \infty}\left(B_{1}\right)}^{2} .
$$

Copyright (c) by SIAM. Unauthorized reproduction of this article is prohibited. 
Using (3.19), (3.24), and Proposition 3, we obtain

$$
\begin{gathered}
\varepsilon^{2} \int_{B_{R / \varepsilon} \backslash B_{2 / \varepsilon}}\left|\hat{W}_{\varepsilon}(k, \cdot)\right|^{2} \leq \varepsilon\|\hat{u}(k, \cdot)\|_{W^{1, \infty}\left(B_{1}\right)}^{2} \quad \text { for } k \geq 1 /(2 \varepsilon), \\
\varepsilon^{2} \int_{B_{R / \varepsilon} \backslash B_{2 / \varepsilon}}\left|\hat{W}_{\varepsilon}(k, \cdot)\right|^{2} \leq \frac{C\left|H_{0}^{(1)}(k)\right|^{2}}{|\ln (\varepsilon k)|^{2}}\|\hat{u}(k, \cdot)\|_{W^{1, \infty}\left(B_{1}\right)}^{2} \quad \text { for } \varepsilon^{\gamma} \leq k \leq 1 /(2 \varepsilon),
\end{gathered}
$$

and

$$
\varepsilon^{2} \int_{B_{R / \varepsilon} \backslash B_{2 / \varepsilon}}\left|\hat{W}_{\varepsilon}(k, \cdot)\right|^{2} \leq \frac{C \varepsilon^{2 \gamma}}{k^{2}} \frac{\left|H_{0}^{(1)}(k)\right|^{2}}{|\ln (\varepsilon k)|^{2}}\|\hat{u}(k, \cdot)\|_{W^{1, \infty}\left(B_{1}\right)}^{2} \quad \text { for } \varepsilon^{5 \gamma / 4} \leq k \leq \varepsilon^{\gamma} .
$$

Using (3.19), (3.24), and Proposition $6,^{5}$ we have

$\varepsilon^{2} \int_{B_{R / \varepsilon} \backslash B_{2 / \varepsilon}}\left|\hat{W}_{\varepsilon}(k, \cdot)\right|^{2} \leq C\left|H_{0}^{(1)}(k)\right|^{2}|\ln (\varepsilon k)|^{2}\|\hat{u}(k, \cdot)\|_{W^{1, \infty}\left(B_{1}\right)}^{2} \quad$ for $0<k \leq \varepsilon^{5 \gamma / 4}$.

In combination with (3.20) and (3.22), this now yields

$$
\begin{aligned}
\varepsilon \int_{0}^{\infty}\left\|\hat{W}_{\varepsilon}(k, \cdot)\right\|_{L^{2}\left(B_{R / \varepsilon} \backslash B_{2 / \varepsilon}\right)} d k \\
=\left(\int_{1 /(2 \varepsilon)}^{\infty}+\int_{\varepsilon^{\gamma}}^{1 /(2 \varepsilon)}+\int_{\varepsilon^{5 \gamma / 4}}^{\varepsilon^{\gamma}}+\int_{0}^{\varepsilon^{5 \gamma / 4}}\right) \varepsilon\left\|\hat{W}_{\varepsilon}(k, \cdot)\right\|_{L^{2}\left(B_{R / \varepsilon} \backslash B_{2 / \varepsilon}\right)} d k \\
\leq C\left(\sqrt{\varepsilon} D a t a+\frac{1}{|\ln \varepsilon|} D a t a+\frac{\varepsilon^{\gamma}}{|\ln \varepsilon|} \int_{\varepsilon^{5 \gamma / 4}}^{\varepsilon^{\gamma}} \frac{|\ln k|}{k}\|\hat{u}(k, \cdot)\|_{W^{1, \infty}} d k\right) \\
\quad+C \int_{0}^{\varepsilon^{5 \gamma / 4}}\left|H_{0}^{(1)}(k)\|\ln (\varepsilon k) \mid\| \hat{u}(k, \cdot) \|_{W^{1, \infty}} d k .\right.
\end{aligned}
$$

Since

$$
\begin{aligned}
\int_{\varepsilon^{5 \gamma / 4}}^{\varepsilon^{\gamma}} \frac{|\ln k|}{k}\|\hat{u}(k, \cdot)\|_{W^{1, \infty}} & \leq\left(\int_{\varepsilon^{5 \gamma / 4}}^{\varepsilon^{\gamma}} \frac{|\ln k|^{2}}{k^{2}}\right)^{1 / 2}\left(\int_{\varepsilon^{5 \gamma / 4}}^{\varepsilon^{\gamma}}\|\hat{u}(k, \cdot)\|_{W^{1, \infty}}^{2}\right)^{1 / 2} \\
& \leq C \varepsilon^{-3 \gamma / 4} \text { Data }
\end{aligned}
$$

and

$$
\begin{aligned}
& \int_{0}^{\varepsilon^{5 \gamma / 4}}\left|H_{0}^{(1)}(k)\|\ln (\varepsilon k) \mid\| \hat{u}(k, \cdot) \|_{W^{1, \infty}}\right. \\
& \quad \leq C\left(\int_{0}^{\varepsilon^{5 \gamma / 4}}|\ln k|^{4}\right)^{1 / 2}\left(\int_{0}^{\varepsilon^{5 \gamma / 4}}\|\hat{u}(k, \cdot)\|_{W^{1, \infty}}^{2}\right)^{1 / 2} \\
& \quad \leq C \varepsilon^{\gamma / 2} \text { Data, }
\end{aligned}
$$

${ }^{5}$ Note that $k<\varepsilon^{5 \gamma / 4}$ ensures that $\varepsilon k<c, \varepsilon k|\ln (\varepsilon k)|^{2}<c \varepsilon^{1+\gamma}$, and $k^{2}|\ln (\varepsilon k)|^{2}<c$ for $\varepsilon$ sufficiently small, as required in Proposition 6.

Copyright $@$ ( ) by SIAM. Unauthorized reproduction of this article is prohibited. 
it follows from (3.25) that

$$
\varepsilon \int_{0}^{\infty}\left\|\hat{W}_{\varepsilon}(k, \cdot)\right\|_{L^{2}\left(B_{R / \varepsilon} \backslash B_{2 / \varepsilon}\right)} d k \leq \operatorname{CData}\left(\sqrt{\varepsilon}+\frac{1}{|\ln \varepsilon|}+\frac{\varepsilon^{\gamma / 4}}{|\ln \varepsilon|}+\varepsilon^{\gamma / 2}\right) .
$$

The fact that $\gamma>0$ now implies

$$
\int_{0}^{\infty} \varepsilon\left\|\hat{W}_{\varepsilon}(k, \cdot)\right\|_{L^{2}\left(B_{R / \varepsilon} \backslash B_{2 / \varepsilon}\right)} d k \leq \frac{C}{|\ln \varepsilon|} \text { Data }
$$

which by a change of variables becomes

$$
\int_{0}^{\infty}\left\|\hat{w}_{\varepsilon}(k, \cdot)\right\|_{L^{2}\left(B_{R} \backslash B_{2}\right)} d k \leq \frac{C}{|\ln \varepsilon|} \text { Data. }
$$

It follows that $\hat{w}_{\varepsilon}(\cdot, x) \in L^{1}\left(\mathbb{R}_{+}\right)$for almost all $x$, and by the inversion formula

$$
w_{\varepsilon}(t, x)=2 \Re\left\{\frac{1}{\sqrt{2 \pi}} \int_{0}^{\infty} \hat{w}_{\varepsilon}(k, x) e^{-i k t} d k\right\}
$$

we obtain (3.16).

Appendix A. The outgoing radiation condition. Suppose $A(x)$ is a real, symmetric matrix valued function, and $\Sigma_{1}, \Sigma_{2}$ are two real functions defined on $\mathbb{R}^{d}$ such that

$$
|\xi|^{2} / \Lambda \leq\langle A(x) \xi, \xi\rangle \leq \Lambda|\xi|^{2}, \quad 1 / \Lambda<\Sigma_{1}(x)<\Lambda, \quad \text { and } \quad 0 \leq \Sigma_{2}(x)<\Lambda
$$

for some positive number $\Lambda$. Furthermore suppose $A, \Sigma_{1}, \Sigma_{2}=I, 1,0$ outside a bounded domain. Given any $f \in L^{2}\left(\mathbb{R}_{+} \times \mathbb{R}^{d}\right)$ such that $\operatorname{supp} f \subset \mathbb{R}_{+} \times K$ for some compact set $K$ of $\mathbb{R}^{d}$, let $v$ be the unique solution of

$$
\Sigma_{1}(x) \partial_{t t}^{2} v(t, x)-\operatorname{div}(A(x) \nabla v(t, x))+\Sigma_{2}(x) \partial_{t} v(t, x)=f(t, x)
$$

with

$$
v(0, x)=\partial_{t} v(0, x)=0 .
$$

We first recall the following classic result, which is a direct consequence of an energy estimate.

Lemma A1. Let $A(x)$ be a real, symmetric matrix valued function, and let $\Sigma_{1}, \Sigma_{2}$ be two real functions defined on $\mathbb{R}^{d}$ such that (A1) holds for some $\Lambda>0$. Given any $f \in L^{2}\left(\mathbb{R}_{+} \times \mathbb{R}^{d}\right)$, let $v$ be the unique solution of (A2) and (A3). We have

$$
\left\|\partial_{t} v(t, \cdot)\right\|_{L^{2}\left(\mathbb{R}^{d}\right)}^{2}+\|\nabla v(t, \cdot)\|_{L^{2}\left(\mathbb{R}^{d}\right)}^{2} \leq C t \int_{0}^{t} \int_{\mathbb{R}^{d}}|f(s, x)|^{2} d x d s \quad t>0
$$

and

$$
\|v(t, \cdot)\|_{L^{2}\left(\mathbb{R}^{d}\right)}^{2} \leq C t^{3} \int_{0}^{t} \int_{\mathbb{R}^{d}}|f(s, x)|^{2} d x d s, \quad t>0 .
$$

Here $C$ denotes a positive constant depending only on $\Lambda$.

Copyright (c) by SIAM. Unauthorized reproduction of this article is prohibited. 
Proof. Multiplying (A2) by $\partial_{t} v$ and integrating the obtained expression over $\mathbb{R}^{d}$, we have

$$
\frac{1}{2} \frac{d}{d t}\left(\int_{\mathbb{R}^{d}} \Sigma_{1}\left|\partial_{t} v\right|^{2}+\langle A \nabla v, \nabla v\rangle\right)+\int_{\mathbb{R}^{d}} \Sigma_{2}\left|\partial_{t} v\right|^{2}=\int_{\mathbb{R}^{d}} f \partial_{t} v .
$$

It follows from (A1) and (A3) that

$$
\left\|\partial_{t} v(t, \cdot)\right\|_{L^{2}\left(\mathbb{R}^{d}\right)}^{2}+\|\nabla v(t, \cdot)\|_{L^{2}\left(\mathbb{R}^{d}\right)}^{2} \leq C\left(\int_{0}^{t}\left(\int_{\mathbb{R}^{d}}|f(x, s)|^{2} d x\right)^{1 / 2} d s\right)^{2}, \quad t>0,
$$

which implies

$$
\left\|\partial_{t} v(t, \cdot)\right\|_{L^{2}\left(\mathbb{R}^{d}\right)}^{2}+\|\nabla v(t, \cdot)\|_{L^{2}\left(\mathbb{R}^{d}\right)}^{2} \leq C t\|f\|_{L^{2}\left([0, t] \times \mathbb{R}^{d}\right)}^{2}, \quad t>0
$$

for some positive constant $C$ depending only on $\Lambda$. This completes the proof of the first inequality. The second inequality follows immediately from the first one and (A3).

We extend $v$ by zero for $t<0$. As a consequence of the preceeding lemma, $v$ is a tempered distribution for a.e. $x \in \mathbb{R}^{d}$. Hence we can as usual define the Fourier transform $\hat{v}$ of $v$ (with respect to $t$ ) by the formula

$$
\int_{-\infty}^{\infty} \hat{v}(k, x) \bar{\phi}(k) d k=\int_{0}^{\infty} v(t, x) \bar{\phi}(t) d t \quad \text { for any } \phi \in \mathcal{S}(\mathbb{R}) .
$$

Here

$$
\check{\phi}(t)=\frac{1}{\sqrt{2 \pi}} \int_{-\infty}^{\infty} \phi(k) e^{-i k t} d k
$$

denotes the inverse of the classical Fourier transform

$$
\hat{\phi}(k)=\frac{1}{\sqrt{2 \pi}} \int_{-\infty}^{\infty} \phi(t) e^{i k t} d t .
$$

In this appendix we show that the Fourier transform $\hat{v}$ is indeed a function and that the corresponding functions $\hat{v}(k, \cdot)$ for almost all $k>0$ are outgoing solutions to the Helmholtz equation; in other words, they are solutions to the Helmholtz equation and they satisfy the outgoing radiation condition

$$
\frac{\partial}{\partial r} \hat{v}(k, \cdot)-i k \hat{v}(k, \cdot)=o\left(r^{-\frac{d-1}{2}}\right) .
$$

Theorem A1. Let $A(x)$ be a real, symmetric matrix valued function and let $\Sigma_{1}, \Sigma_{2}$ be two real functions defined on $\mathbb{R}^{d}$ such that (A1) holds for some $\Lambda>0$, and $A, \Sigma_{1}, \Sigma_{2}=I, 1,0$ outside a bounded domain. Given any $f \in L^{2}\left(\mathbb{R}_{+} \times \mathbb{R}^{d}\right)$ such that supp $f \subset \mathbb{R}_{+} \times K$ for some compact subset $K$ of $\mathbb{R}^{d}$, let $v$ be the unique solution of (A2) and (A3). For almost all $k>0, \hat{v}(k, \cdot) \in H_{\text {loc }}^{1}\left(\mathbb{R}^{d}\right)$ is the unique outgoing solution to the equation

$$
\operatorname{div}(A(x) \nabla \hat{v}(k, x))+k^{2} \Sigma_{1}(x) \hat{v}(k, x)+i k \Sigma_{2}(x) \hat{v}(k, x)=-\hat{f}(k, x)
$$

with $\hat{f}(k, x)$ denoting the Fourier transform of $f(t, x)$ (extended by zero for negative time). 
Proof. Let $v_{\varepsilon}$ be the unique solution to

$$
\Sigma_{1}(x) \partial_{t t}^{2} v_{\varepsilon}(t, x)-\operatorname{div}\left(A(x) \nabla v_{\varepsilon}(t, x)\right)+\left(\Sigma_{2}(x)+\varepsilon\right) \partial_{t} v_{\varepsilon}(t, x)=f(t, x)
$$

with $v_{\varepsilon}(0, x)=\partial_{t} v_{\varepsilon}(0, x)=0$. From the analogue of (A4), we conclude that

$$
\varepsilon^{2} \int_{0}^{t}\left\|\partial_{s} v_{\varepsilon}(s, \cdot)\right\|_{L^{2}\left(\mathbb{R}^{d}\right)}^{2} d s \leq C,
$$

where $C$ denotes a positive constant depending only on $\|f\|_{L^{2}\left(\mathbb{R}_{+} \times \mathbb{R}^{d}\right)}$ and $\Lambda$. Set

$$
w_{\varepsilon}=v_{\varepsilon}-v .
$$

We have

$$
\Sigma_{1}(x) \partial_{t t}^{2} w_{\varepsilon}(t, x)-\operatorname{div}\left(A(x) \nabla w_{\varepsilon}(t, x)\right)+\left(\Sigma_{2}(x)+\varepsilon\right) \partial_{t} w_{\varepsilon}(t, x)=-\varepsilon \partial_{t} v
$$

with $w_{\varepsilon}(0, x)=\partial_{t} w_{\varepsilon}(0, x)=0$. It follows immediately from Lemma A1 that

$$
v_{\varepsilon} \rightarrow v \quad \text { in } \quad L^{2}\left((0, T) \times \mathbb{R}^{d}\right) \quad \text { as } \quad \varepsilon \rightarrow 0 \quad \text { for any } T>0 .
$$

Let $\hat{v}_{\varepsilon}$ denote the Fourier transform of $v_{\varepsilon}$. From Plancherel's theorem and the energy estimate (A7) we have

$$
\int_{\mathbb{R}} k^{2}\left\|\hat{v}_{\varepsilon}(k, \cdot)\right\|_{L^{2}\left(\mathbb{R}^{d}\right)}^{2} d k<\infty .
$$

In other words $\hat{v}_{\varepsilon}(k, \cdot) \in L^{2}\left(\mathbb{R}^{d}\right)$ for almost all $k$. It is straightforward to check that $\hat{v}_{\varepsilon}(k, \cdot)$ satisfies the equation

$$
\operatorname{div}\left(A(x) \nabla \hat{v}_{\varepsilon}(k, x)\right)+k^{2} \Sigma_{1}(x) \hat{v}_{\varepsilon}(k, x)+i k \Sigma_{2}(x) \hat{v}_{\varepsilon}(k, x)+i k \varepsilon \hat{v}_{\varepsilon}(k, x)=-\hat{f}(k, x) .
$$

As a consequence $\hat{v}_{\varepsilon}(k, \cdot)$ lies in $H^{1}\left(\mathbb{R}^{d}\right)$ for almost all $k$, and it is the unique solution to (A10) in this space. By the limiting absorption principle (see, e.g., [10, section 4.6]) we have for $k>0$,

$$
\hat{v}_{\varepsilon}(k, \cdot) \rightarrow \hat{V}(k, \cdot) \text { weakly in } H_{l o c}^{1}\left(\mathbb{R}^{d}\right),
$$

where $\hat{V}(k, x) \in H_{l o c}^{1}\left(\mathbb{R}^{d}\right)$ is the unique outgoing solution to

$$
\operatorname{div}(A(x) \nabla \hat{V}(k, x))+k^{2} \Sigma_{1}(x) \hat{V}(k, x)+i k \Sigma_{2}(x) \hat{V}(k, x)=-\hat{f}(k, x) .
$$

From (A9) and (A11) it follows that

$$
\hat{v}_{\varepsilon}(k, x) \text { converges to } \hat{V}(k, x) \text { in the distributional sense on } \mathbb{R}_{+} \times \mathbb{R}^{d} \text {. }
$$

On the other hand, let $\phi(k, x)$ be a $C^{\infty}$ test function (in $k$ and $x$ ) with compact support; then

$$
\begin{aligned}
\int_{-\infty}^{\infty} & \int_{\mathbb{R}^{d}}\left(\hat{v}_{\varepsilon}(k, x)-\hat{v}(k, x)\right) \bar{\phi}(k, x) d x d k \\
= & \int_{0}^{\infty} \int_{\mathbb{R}^{d}}\left(v_{\varepsilon}(t, x)-v(t, x)\right) \bar{\phi}(t, x) d x d t \\
= & \int_{0}^{T} \int_{\mathbb{R}^{d}}\left(v_{\varepsilon}(t, x)-v(t, x)\right) \bar{\phi}(t, x) d x d t \\
& \quad+\int_{T}^{\infty} \int_{\mathbb{R}^{d}}\left(v_{\varepsilon}(t, x)-v(t, x)\right) \bar{\phi}(t, x) d x d t
\end{aligned}
$$

Copyright $@$ by SIAM. Unauthorized reproduction of this article is prohibited. 
Since $\operatorname{supp} \check{\phi}(t, x) \subset \mathbb{R} \times B_{R}$ for some $R>0$, it follows from Lemma A1 that

$$
\int_{T}^{\infty} \int_{\mathbb{R}^{d}}\left(v_{\varepsilon}(t, x)-v(t, x)\right) \bar{\phi}(t, x) d x d t \leq C \int_{T}^{\infty} t^{3 / 2}\|\check{\phi}(t, \cdot)\|_{L^{2}\left(\mathbb{R}^{d}\right)} d t .
$$

Since $\|\check{\phi}(t, \cdot)\|_{L^{2}\left(\mathbb{R}^{d}\right)}$ decreases faster than any negative power of $t$, it follows that given any $\delta>0$, we may choose $T$ such that

$$
\left|\int_{T}^{\infty} \int_{\mathbb{R}^{d}}\left(v_{\varepsilon}(t, x)-v(t, x)\right) \bar{\phi}(t, x) d x d t\right|<\delta / 2 \quad \forall 0<\varepsilon<1 .
$$

Since $v_{\varepsilon}$ converges to $v$ in $L^{2}\left((0, T) \times \mathbb{R}^{d}\right)$, according to (A8), we may now choose $\varepsilon$ sufficiently small that

$$
\left|\int_{0}^{T} \int_{\mathbb{R}^{d}}\left(v_{\varepsilon}(t, x)-v(t, x)\right) \check{\phi}(t, x) d x d t\right|<\delta / 2 .
$$

A combination of these two estimates with (A13) yields that

$\hat{v}_{\varepsilon}$ converges to $\hat{v}$ in the distributional sense (with respect to $k$ and $x$ ).

As a consequence of this and (A12) we conclude that $\hat{v}(k, \cdot)=\hat{V}(k, \cdot)$ for almost all $k>0$, which completes the proof of Theorem A1.

Using the same technique, we can also prove the following.

TheOREM A2. Let $D$ be a smooth, bounded, open subset of $\mathbb{R}^{d}$. Let $f \in L^{2}\left(\mathbb{R}_{+} \times\right.$ $\left.\left(\mathbb{R}^{d} \backslash D\right)\right)$ be such that $\operatorname{supp} f \in \mathbb{R}_{+} \times K$ for some compact subset $K$ of $\mathbb{R}^{d} \backslash D$. Suppose $A(x)$ is a real, symmetric matrix valued function and $\Sigma_{1}, \Sigma_{2}$ are two real functions defined on $\mathbb{R}^{d} \backslash D$ such that (A1) holds for some $\Lambda>0$. Suppose also $A, \Sigma_{1}, \Sigma_{2}=I, 1,0$ outside a bounded domain and let $v$ be the unique solution to the equation

(A14)

$$
\left\{\begin{array}{l}
\Sigma_{1}(x) \partial_{t t}^{2} v(t, x)-\operatorname{div}(A(x) \nabla v(t, x))+\Sigma_{2}(x) \partial_{t} v(t, x)=f(t, x) \quad \text { in } \mathbb{R}_{+} \times\left(\mathbb{R}^{d} \backslash \bar{D}\right), \\
v=0 \quad \text { on } \mathbb{R}_{+} \times \partial D, \\
v(t=0)=\partial_{t} v(t=0)=0 .
\end{array}\right.
$$

Let $\hat{v}(k, x)$ denote the Fourier transform of $v(t, x)$ with respect to $t$. Then $\hat{v}(k, \cdot) \in$ $H_{\text {loc }}^{1}\left(\mathbb{R}^{d} \backslash D\right)$ is the unique outgoing solution to

$$
\operatorname{div}(A \nabla \hat{v}(k, x))+k^{2} \Sigma_{1} \hat{v}(k, x)+i k \Sigma_{2} \hat{v}(k, x)=-\hat{f}(k, x) \quad \text { in } \mathbb{R}^{d} \backslash \bar{D}
$$

for almost all $k>0$.

Appendix B. Decay of solutions of the 2d wave equation. In this section, we establish the decay of solutions of the $2 \mathrm{~d}$ wave equation which is an ingredient in the proof of Theorem 4 .

TheOREM B1. Let $f(t, x), u_{0}(x)$, and $u_{1}(x)$ be smooth functions such that $\operatorname{supp} f \subset[0,1] \times\left(B_{4} \backslash B_{2}\right)$ and $\operatorname{supp} u_{0}, \operatorname{supp} u_{1} \subset B_{4} \backslash B_{2}$. Let $u$ be the unique solution of the system

$$
\begin{cases}\partial_{t t}^{2} u-\Delta u=f & \text { in } \mathbb{R}_{+} \times \mathbb{R}^{2}, \\ u(t=0)=u_{0} & \text { in } \mathbb{R}^{2}, \\ \partial_{t} u(t=0)=u_{1} & \text { in } \mathbb{R}^{2} .\end{cases}
$$

Copyright $@$ by SIAM. Unauthorized reproduction of this article is prohibited. 
There exist a positive constant $C$ and an integer $m>0$ such that

$$
\int_{0}^{\infty}\|u(t, \cdot)\|_{L^{2}\left(B_{1}\right)}^{2} d t \leq C \operatorname{Data}^{2}
$$

where Data $=\|f\|+\left\|u_{0}\right\|+\left\|u_{1}\right\|$ and $\|\cdot\|$ denotes the $C^{m}$-norm.

Proof. The theorem follows immediately from the explicit formula for solutions of the wave equation in $2 \mathrm{~d}$. For the convenience of the reader, we present the proof. Let $v$ and $w$ be the unique solutions to the systems

$$
\begin{cases}\partial_{t t}^{2} v-\Delta v=0 & \text { in } \mathbb{R}_{+} \times \mathbb{R}^{2} \\ v(t=0)=u_{0} & \text { in } \mathbb{R}^{2} \\ \partial_{t} v(t=0)=u_{1} & \text { in } \mathbb{R}^{2}\end{cases}
$$

and

$$
\begin{cases}\partial_{t t}^{2} w-\Delta w=f & \text { in } \mathbb{R}_{+} \times \mathbb{R}^{2} \\ w(t=0)=0 & \text { in } \mathbb{R}^{2} \\ \partial_{t} w(t=0)=0 & \text { in } \mathbb{R}^{2}\end{cases}
$$

respectively. It is clear that

$$
u=v+w .
$$

We have

$$
v(t, x)=\frac{1}{2} f_{B(x, t)} \frac{t u_{0}(y)+t^{2} u_{1}(y)+t\left\langle\nabla u_{0}(y), y-x\right\rangle}{\left(t^{2}-|y-x|^{2}\right)^{1 / 2}} d y,
$$

which implies

$$
|v(t, x)| \leq C\left(\frac{1}{t^{2}}\left\|u_{0}\right\|+\frac{1}{t}\left\|u_{1}\right\|\right) \quad \forall x \in B_{1}, t>6 .
$$

Direct integration therefore yields

$$
\int_{6}^{\infty}\|v(t, \cdot)\|_{L^{2}\left(B_{1}\right)}^{2} d t \leq C \operatorname{Data}^{2} .
$$

By a standard energy estimate

$$
\int_{0}^{6}\|v(t, \cdot)\|_{L^{2}\left(B_{1}\right)}^{2} d t \leq C D_{a t a}^{2}
$$

a combination with (B2) now gives

$$
\int_{0}^{\infty}\|v(t, \cdot)\|_{L^{2}\left(B_{1}\right)}^{2} d t \leq C D a t a^{2}
$$

On the other hand, we have

$$
w(t, x)=\int_{0}^{t} w(t, x ; s) d s,
$$

Copyright $@$ by SIAM. Unauthorized reproduction of this article is prohibited. 
where $w(t, x ; s)$ is the unique solution to the equation

$$
\begin{cases}\partial_{t t}^{2} w(t, x ; s)-\Delta w(t, x ; s)=0 & \text { in }(s,+\infty) \times \mathbb{R}^{2}, \\ w(t=s, x ; s)=0 & \text { in } \mathbb{R}^{2}, \\ \partial_{t} w(t=s, x ; s)=f(s, x) & \text { in } \mathbb{R}^{2} .\end{cases}
$$

The explicit formula for solutions to the wave equation gives

$$
w(t, x ; s)=\frac{1}{2} f_{B(x, t-s)} \frac{(t-s)^{2} f(s, y)}{\left((t-s)^{2}-|y-x|^{2}\right)^{1 / 2}} d y, \quad t>s>0 .
$$

For $x \in B_{1}$ this implies

$$
\begin{aligned}
w(t, x ; s) & =0 \quad \text { for } s>1, t>s>0 \quad \text { and } \\
|w(x, t, s)| & \leq C t^{-1} \text { Data } \quad \text { for } s<1, t>7 .
\end{aligned}
$$

Combining (B4), (B5), and the standard energy estimate for $w(t, x ; s)$, we obtain

$$
\int_{0}^{\infty}\|w(t, \cdot)\|_{L^{2}\left(B_{1}\right)}^{2} d t \leq C \operatorname{Data}^{2} .
$$

The estimate of Theorem B1 follows from (B1), (B3), and (B6).

Remark 6. Derivatives of $u$ with respect to $x$ or $t$ satisfy an initial value problem similar to that satisfied by $u$, with the data replaced by its appropriate derivatives (and combinations thereof). It thus follows immediately from Theorem B1 that

$$
\int_{0}^{\infty}\left(\|u(t, \cdot)\|_{H^{3}\left(B_{1}\right)}^{2}+\left\|\partial_{t} u(t, \cdot)\right\|_{H^{3}\left(B_{1}\right)}^{2}\right) d t \leq C \operatorname{Data}^{2}
$$

where Data $=\|f\|+\left\|u_{0}\right\|+\left\|u_{1}\right\|$, and $\|\cdot\|$ denotes some appropriate $C^{m}$-norm.

\section{REFERENCES}

[1] W. Cai, U. K. Chettiar, A. V. Kildishev, and V. M. Shalaev, Optical cloaking with metamaterials, Nature Photonics, 1 (2007), pp. 224-227.

[2] A. Greenleaf, Y. Kurylev, M. Lassas, and G. Uhlmann, Full-wave invisibility of active devices at all frequencies, Comm. Math. Phys., 275 (2007), pp. 749-789.

[3] A. Greenleaf, Y. Kurylev, M. Lassas, and G. Uhlmann, Improvement of cylindrical cloaking with the SHS lining, Optics Express, 15 (2007), pp. 12717-12734.

[4] A. Greenleaf, Y. Kurylev, M. Lassas, and G. Uhlmann, Invisibility and inverse problems, Bull. Amer. Math. Soc., 46 (2009), pp. 55-97.

[5] A. Greenleaf, M. Lassas, and G. Uhlmann, On nonuniqueness for Calderon's inverse problem, Math. Res. Lett., 10 (2003), pp. 685-693.

[6] H. Haddar, P. Joly, and H.-M. NGuYen, Generalized impedance boundary conditions for scattering by strongly absorbing obstacles: The scalar case, Math. Models Methods Appl. Sci., 15 (2005), pp. 1273-1300.

[7] V. V. Jikov, S. M. Kozlov, And O. A. Oleinik, Homogenization of Differential Operators and Integral Functionals, Springer-Verlag, Berlin, 1994.

[8] R. V. Kohn, D. Onofrei, M. S. Vogelius, and M. I. Weinstein, Cloaking via change of variables for the Helmholtz equation, Comm. Pure Appl. Math., 63 (2010), pp. 973-1016.

[9] R. V. Kohn, H. Shen, M. S. Vogelius, And M. I. Weinstein, Cloaking via change of variables in electric impedance tomography, Inverse Problems, 24 (2008), 015016.

[10] R. LeIs, Initial-boundary value problems in mathematical physics, B. G. Teubner, Stuttgart, 1986.

[11] U. Leonhardt, Optical conformal mapping, Science, 312 (2006), pp. 1777-1780. 
[12] H. Liu, Virtual reshaping and invisibility in obstacle scattering, Inverse Problems, 25 (2009), 045006.

[13] C. S. Morawetz And D. Ludwig, An inequality for the reduced wave operator and the justification of geometrical optics, Comm. Pure Appl. Math., 21 (1968), pp. 187-203.

[14] F. Murat and L. Tartar, $H$-convergence, in Topics in the Mathematical Modelling of Composite Materials, A Cherkaev and R. Kohn, eds., Progr. Nonlinear Differential Equations Appl., Birkhäuser Boston, Cambridge, MA, 1997.

[15] H.-M. NGuyen, Cloaking for the Helmholtz equation in the whole space, Comm. Pure Appl. Math., 63 (2010), pp. 1505-1524.

[16] H.-M. NGUYEN, Approximate cloaking for the Helmholtz equation via transformation optics and consequences for perfect cloaking, Comm. Pure Appl. Math., 65 (2012), pp. 155-188.

[17] H.-M. NGuYen And M. S. Vogelius, Full range scattering estimates and their application to cloaking, Arch. Ration. Mech. Anal., 203 (2011), pp. 769-807.

[18] J. B. Pendry, D. Schurig, And D. R. Smith, Controlling electromagnetic fields, Science, 312 (2006), pp. 1780-1782.

[19] Z. Ruan, M. Yan, C. M. NefF, And M. Qiu, Ideal cylindrical cloak: Perfect but sensitive to tiny perturbations, Phys. Rev. Lett., 99 (2007), 113903.

[20] D. Schurig, J. J. Mock, J. Justice, S. A. Cummer, J. B. Pendry, A. F. Starr, and D. R. Sмiтh, Metamaterial electromagnetic cloak at microwave frequencies, Science, 314 (2006), 1133628.

[21] G. N. Watson, A Treatise on the Theory of Bessel Functions, Cambridge University Press, Cambridge, UK, 1944.

[22] R. Weder, A rigorous analysis of high-order electromagnetic invisibility cloaks, J. Phys. A, 41 (2008), 065207

[23] B. Wood, Metamaterials and invisibility, C. R. Physique, 10 (2009), pp. 379-390.

[24] M. YAN, Z. RUAN, AND M. QIU, Cylindrical invisibility cloak with simplified material parameters is inherently visible, Phys. Rev. Lett., 99 (2007), 233901.

Copyright (c) by SIAM. Unauthorized reproduction of this article is prohibited. 